\title{
HIRZEBRUCH-RIEMANN-ROCH FORMULAE ON IRREDUCIBLE SYMPLECTIC KÄHLER MANIFOLDS
}

\author{
MICHAEL BRITZE AND MARC A. NIEPER
}

\begin{abstract}
In this article we investigate Hirzebruch-Riemann-Roch formulae for line bundles on irreducible symplectic Kähler manifolds. As Huybrechts has shown in [1], for every irreducible complex Kähler manifold $X$ of dimension $2 n$, there are numbers $a_{0}, a_{2}, \ldots, a_{2 n}$ such that $\chi(L)=\sum_{k=0}^{n} a_{2 k} /(2 k) ! q_{X}\left(c_{1}(L)\right)^{k}$ for the Euler characteristic of a line bundle $L$, where $q_{X}: \mathrm{H}^{2}(X, \mathbb{C}) \rightarrow \mathbb{C}$ is the Beauville-Bogomolov quadratic form of $X$.

Using Rozansky-Witten classes similar to Hitchin and Sawon in 10], we obtain a formula expressing the $a_{2 k}$ in terms of Chern numbers of $X$. Furthermore, for the $n$-th generalized Kummer variety $K^{n} A$ (see [3]), we prove $\chi(L)=(n+1)\left(\begin{array}{c}q\left(c_{1}(L)\right) / 2+n \\ n\end{array}\right)$ by purely algebro-geometric methods, where $q$ is the form $q_{X}$ up to a positive rational constant. A similar formula is already known for the Hilbert scheme of zero-dimensional subschemes of length $n$ on a K3-surface (c.f. 何).

Using our results, we are able to calculate all Chern numbers of the generalized Kummer varieties $K^{n} A$ for $n \leq 5$. For $n \leq 4$ these results were previously obtained by Sawon (c.f. [16]).
\end{abstract}

\section{Contents}

1. Some linear algebra 2

2. Graph homology 3

2.1. The graph homology space 3

2.2. Operations with graphs and special graphs 5

\begin{tabular}{ll|l}
$2.3 . \quad \Omega$ as an eigenvector of the operator $\partial$ & 7
\end{tabular}

\begin{tabular}{lr|r|}
\hline 3. & Rozansky-Witten classes & 9
\end{tabular}

$\begin{array}{lll}3.1 . & \text { Rozansky-Witten classes in genera } & 10\end{array}$

3.2. $\quad$ Rozansky-Witten invariants of symplectic Kähler manifolds 11

\begin{tabular}{lll|l}
3.3. & Examples of Rozansky-Witten classes & 12
\end{tabular}

3.4. Properties of Rozansky-Witten classes 13

4. The Euler characteristic of a line bundle in terms of the quadratic form 13

\begin{tabular}{llll}
\hline 4.1. & The quadratic form on an irreducible symplectic manifold & 13
\end{tabular}

\begin{tabular}{ll|l}
$4.2 . \quad$ A Hirzebruch-Riemann-Roch formula & 14
\end{tabular}

\begin{tabular}{ll|l}
\hline 5. Generalized Kummer Varieties. & 17
\end{tabular}

\begin{tabular}{ll|l} 
5.1. Basic Facts & 17
\end{tabular}

5.2. $\quad$ Explicit Hirzebruch-Riemann-Roch for $K^{n} A . \quad 18$

\begin{tabular}{ll|l}
\hline 6. & Characteristic numbers of $K^{n} A$ & 22
\end{tabular}

References 23

We are very grateful to Daniel Huybrechts for having carefully read preliminary versions of this paper and to Daniel Huybrechts, Manfred Lehn and many others for their support to us and helpful discussions about the subject. 


\section{Some LineAr ALGEBra}

In this section, let $k$ denote a field of characteristic zero, $V$ a $k$-vector space and $A$ a $k$-algebra.

We identify the exterior algebra $\bigwedge\left(V^{*}\right) \otimes_{k} A$ with the space $\bigoplus_{r=0}^{n} \operatorname{Alt}^{r}(V, A)$ of alternating multilinear forms on $V$ with values in $A$ as vector spaces by setting

$$
\left(\left(\alpha_{1} \wedge \cdots \wedge \alpha_{r}\right) \otimes a\right)\left(v_{1}, \ldots, v_{r}\right)=\operatorname{det}\left(\left(\alpha_{i}\left(v_{j}\right)\right)_{i j}\right) \cdot a
$$

for $\alpha_{1}, \ldots, \alpha_{r} \in V^{*}, v_{1}, \ldots, v_{r} \in V$ and $a \in A$.

Definition 1. We call an element $\sigma \in \bigwedge^{2}\left(V^{*}\right)$ a symplectic form on $V$ and $V$ together with $\sigma$ a symplectic $k$-vector space if the map

$$
I_{\sigma}: V \rightarrow V^{*}, v \mapsto \sigma(\cdot, v)
$$

is an isomorphism of vector spaces.

Remark. A symplectic vector space is always of even dimension.

If $\sigma$ is a fixed symplectic form on $V$, we will identify $V$ and $V^{*}$ using the isomorphism $I_{\sigma}$. In particular, we have an induced dual symplectic form $\tilde{\sigma} \in \bigwedge^{2} V$ on $V^{*}$.

For the rest of this section, let $\sigma$ be a fixed symplectic form on a $2 n$-dimensional vector space $V$.

Definition 2. We define a non-singular pairing

$$
\langle\cdot, \cdot\rangle:\left(\bigwedge V^{*} \otimes_{k} A\right) \otimes_{k}\left(\bigwedge V^{*} \otimes_{k} A\right) \rightarrow A
$$

by setting

$$
\left\langle\left(\alpha_{1} \wedge \cdots \wedge \alpha_{r}\right) \otimes a,\left(\beta_{1} \wedge \cdots \wedge \beta_{s}\right) \otimes b\right\rangle=\delta_{r s} \operatorname{det}\left(\left(\sigma^{*}\left(\alpha_{i}, \beta_{j}\right)\right)_{i j}\right) \cdot a b
$$

for $\alpha_{1}, \ldots, \alpha_{r}, \beta_{1}, \ldots, \beta_{s} \in V^{*}$ and $a, b \in A$, where $\delta_{r s}$ is Kronecker's $\delta$.

The following proposition will be used later in the case that $V$ is a tangent space of a complex manifold at a point $p$ and $A$ is the algebra of anti-holomorphic forms at $p$.

Proposition 1. Let $\sigma$ be a symplectic form on $V$ and $\int: \bigwedge\left(V^{*}\right) \otimes_{k} A \rightarrow \bigwedge^{2 n}\left(V^{*}\right) \otimes_{k}$ $A$ the canonical projection onto the forms of top degree.

For every $\alpha \in \bigwedge\left(V^{*}\right)$ we have

$$
\int(\alpha \wedge \exp \sigma)=\int(\langle\alpha, \exp \sigma\rangle \exp \sigma) .
$$

Proof. We can assume that $A=k$ and $\alpha=\alpha_{1} \wedge \cdots \wedge \alpha_{2 p} \in \bigwedge^{2 p}\left(V^{*}\right)$ with $p \in \mathbb{N}_{0}$, $\alpha_{i} \in V^{*}$. So we have to prove

$$
\alpha^{2 p} \wedge \sigma^{(n-p)}=\frac{(n-p) !}{p ! \cdot n !}\left\langle\alpha, \sigma^{p}\right\rangle \sigma^{n} .
$$

Let $e_{1}, \ldots, e_{2 n}$ be a symplectic basis of $V$ and $\vartheta^{1}, \ldots, \vartheta^{2 n}$ the corresponding dual basis of $V^{*}$, i.e. $\sigma=\sum_{i=1}^{n} \vartheta^{2 i-1} \wedge \vartheta^{2 i}$ and $\tilde{\sigma}=\sum_{i=1}^{n} e_{2 i-1} \wedge e_{2 i}$. It follows that 


$$
\begin{aligned}
\sigma^{n} & =n ! \cdot \vartheta^{1} \wedge \cdots \wedge \vartheta^{2 n} . \text { It is } \\
& \left\langle\alpha \wedge \sigma^{(n-p)}, \sigma^{n}\right\rangle \\
& =n !(n-p) ! \sum_{1 \leq i_{1}<\cdots<i_{n-p} \leq n} \begin{array}{r}
\left\langle\alpha_{1} \wedge \cdots \wedge \alpha_{2 p} \wedge \vartheta^{2 i_{1}-1} \wedge \vartheta^{2 i_{i}} \wedge \cdots\right. \\
\cdots
\end{array} \\
& =n !(n-p) ! \sum_{1 \leq j_{1}<\cdots<j_{p} \leq n}\left\langle\alpha_{1} \wedge \cdots \wedge \alpha_{2 p}, \vartheta^{2 j_{1}-1} \wedge \vartheta^{2 i_{n}-p}, \vartheta^{1} \wedge \cdots \wedge \vartheta^{2 n}\right\rangle \\
& =\frac{n !(n-p) !}{p !}\left\langle\alpha, \sigma^{p}\right\rangle .
\end{aligned}
$$

Since $\sigma^{n}$ spans $\bigwedge^{2 n}\left(V^{*}\right)$ and $\left\langle\sigma^{n}, \sigma^{n}\right\rangle=n !^{2}$, this proves the proposition.

Remark. Hitchin and Sawon have stated this result for $\alpha$ being a two-form in [10, p. 8. Note that they identify the exterior algebra over $V^{*}$ with the alternating forms on $V$ in a different way than we do.

Extending their formula to arbitrary degree of $\alpha$ is crucial for this work.

Definition 3. For every set $S$, we denote by $\bigwedge_{k} S$ the Graßmann algebra generated by the elements of $S$ over $k$. If $S^{\prime}$ is a subset of $S$, we view $\bigwedge_{k}\left(S^{\prime}\right)$ canonically as a subalgebra of $\bigwedge_{k} S$. We denote by $\operatorname{Sym}_{k}^{n} S$ the $n$-th symmetric product of the $k$-vector space spanned by the elements of $S$.

\section{Graph homology}

2.1. The graph homology space. In this article, graph means a collection of vertices connected by edges, i.e. every edge connects two vertices. We want to call a half-edge (i.e. an edge together with an adjacent vertex) of a graph a flag. So, every edge consists of exactly two flags. Every flag belongs to exactly one vertex of the graph. On the other hand, a vertex is given by the set of its flags. It is called univalent, if there is only one flag belonging to it, and it is called trivalent, if there are exactly three flags belonging to it. We shall identify edges and vertices with the set of their flags.

A graph is called vertex-oriented if, for every vertex, a cyclic ordering of its flags is fixed.

Definition 4. A Jacobi diagram is a vertex-oriented graph with only uni- and trivalent vertices. A connected Jacobi diagram is a Jacobi diagram which is connected as a graph. A trivalent Jacobi diagram is a Jacobi diagram with no univalent vertices.

We define the degree of a Jacobi diagram to be the number of its vertices. It is always an even number.

Example. The empty graph is a Jacobi diagram, denoted by 1 . The unique Jacobi diagram consisting of two univalent vertices (which are connected by an edge) is denoted by $\ell$.

We can always draw a Jacobi diagram in a planar drawing so that it looks like a planar graph with vertices of valence 1,3 or 4 . Each 4 -valent vertex has to be interpreted as a crossing of two non-connected edges of the drawn graph and not as one of its vertices. Further, we want the counter-clockwise ordering of the flags at each trivalent vertex in the drawing to be the same as the given cyclic ordering.

In drawn Jacobi diagrams, we also use a notation like $\cdots \stackrel{n}{-} \cdots$ for a part of a graph which looks like a long line with $n$ univalent vertices ("legs") attached to it, 
for example $\ldots \perp \perp \ldots$ for $n=3$. The position of $n$ indicates the placement of the legs relative to the "long line".

Definition 5. Let $S$ be a totally ordered set with $n$ elements. We set

$$
\varepsilon(S):=s_{1} \wedge \cdots \wedge s_{n} \in \bigwedge_{\mathbb{Q}} S .
$$

Here, $s_{1}, \ldots, s_{n}$ are the elements of $S$ in increasing order.

Definition 6. Let $S$ be a cyclicly ordered set with an odd number $n$ of elements. We set

$$
\varepsilon(S):=s_{1} \wedge \cdots \wedge s_{n} \in \bigwedge_{\mathbb{Q}} S
$$

Here, $s_{1}, \ldots, s_{n}$ are the elements of $S$ in an order compatible with the given cyclic one. The definition of $\varepsilon(S)$ does not depend on this order as long as the compatibility condition is fulfilled.

Example. If $t$ is a trivalent vertex in a Jacobi diagram, it makes sense to write $\varepsilon(t)$ because we have said that we identify a vertex with the set of the flags belonging to it.

Definition 7. Let $\Gamma$ be a Jacobi diagram with $k$ trivalent and $l$ univalent vertices, so $m:=\frac{3 k+l}{2}$ is the number of its edges. Let $F$ be the set of its flags, $E$ the set of its edges, $T$ the set of its trivalent vertices and $U$ the set of its univalent vertices.

A choice of total orderings of the sets $T, U$ and every set $e \in E$ (recall that an edge is identified with the set of the two flags belonging to it) are said to be compatible with the orientation of $\Gamma$ if the equality

$$
\varepsilon\left(t_{1}\right) \wedge \cdots \wedge \varepsilon\left(t_{k}\right) \wedge \varepsilon\left(u_{1}\right) \wedge \cdots \wedge \varepsilon\left(u_{l}\right)=\varepsilon\left(e_{1}\right) \wedge \cdots \wedge \varepsilon\left(e_{m}\right)
$$

holds in the Graßmann algebra $\bigwedge_{\mathbb{Q}} F$ generated by the elements of $F$. Here, $t_{1}, \ldots, t_{k}$ are the elements of $T$ in increasing order, $u_{1}, \ldots, u_{l}$ are the elements of $U$ in increasing order, and $e_{1}, \ldots, e_{m}$ are all the edges in arbitrary order.

Definition 8. We define $\mathcal{B}$ to be the $\mathbb{Q}$-vector space spanned by all Jacobi diagrams modulo the IHX relation

$$
\Upsilon=H-X
$$

and the anti-symmetry (AS) relation

$$
Y+Y=0,
$$

which can be applied anywhere within a diagram. (For this definition see also [2] and 17].)

Furthermore, let $\mathcal{B}^{\prime}$ be the subspace of $\mathcal{B}$ spanned by all Jacobi diagrams not containing $\ell$ as a component, and let $\mathcal{B}^{\text {t }}$ be the subspace of $\mathcal{B}^{\prime}$ spanned by all trivalent Jacobi diagrams. All these are graded and double-graded. The grading is induced by the degree of the Jacobi diagrams, the double-grading by the number of univalent and trivalent vertices.

The completion of $\mathcal{B}$ (resp. $\mathcal{B}^{\prime}$, resp. $\mathcal{B}^{\mathrm{t}}$ ) with respect to the grading will be denoted by $\hat{\mathcal{B}}$ (resp. $\hat{\mathcal{B}}^{\prime}$, resp. $\hat{\mathcal{B}}^{\mathrm{t}}$ ).

All these spaces are called graph homology spaces and their elements are called graph homology classes or graphs for short.

Remarks. The subspaces $\mathcal{B}_{k}$ of $\hat{\mathcal{B}}$ spanned by the Jacobi diagrams of degree $k$ are always of finite dimension. The subspace $\mathcal{B}_{0}$ is one-dimensional and spanned by the graph homology class of the empty diagram 1. 
Example. If $\gamma$ is a graph which has a part looking like $\cdots \frac{n}{-\cdots}$, it will become $(-1)^{n} \gamma$ if we substitute the part $\cdots \frac{n}{-\cdots}$ by $\cdots-\cdots$ due to the anti-symmetry relation.

\subsection{Operations with graphs and special graphs.}

Definition 9. Disjoint union of Jacobi diagrams induces a bilinear map

$$
\hat{\mathcal{B}} \times \hat{\mathcal{B}} \rightarrow \hat{\mathcal{B}},\left(\gamma, \gamma^{\prime}\right) \mapsto \gamma \cup \gamma^{\prime}
$$

By mapping $1 \in \mathbb{Q}$ to $1 \in \hat{\mathcal{B}}$, the space $\hat{\mathcal{B}}$ becomes a graded $\mathbb{Q}$-algebra, which has no components in odd degrees. Often, we omit the product sign " $\cup$ ". $\mathcal{B}, \mathcal{B}^{\prime}, \mathcal{B}^{t}$ and so on are subalgebras.

Definition 10. Let $k \in \mathbb{N}$. We call the graph homology class of the Jacobi diagram

$\bigcirc^{2 k}$ the $2 k$-wheel $w_{2 k}$, i.e. $w_{2}=-\circ, w_{4}=\propto \alpha$ and so on. It has $2 k$ univalent and $2 k$ trivalent vertices. Further, we set $w_{0}:=1$.

For $k_{1}, k_{2} \in \mathbb{N}$, we call the graph homology class of the Jacobi diagram $\underset{k_{2}}{\ominus} \mathrm{a}$ double-wheel, denoted by $w_{k_{1}, k_{2}}$. In particular, $w_{0,0}=\ominus$.

Next, we define a very special element $\Omega$ of the graph homology space with very remarkable properties, which have been proven by Bar-Natan, Le and Thurston (see [17]).

Definition 11. Let the series $\left(b_{2 k}\right)_{k} \in \mathbb{C}^{\mathbb{N}_{0}}$ of the modified Bernoulli numbers (c.f. [17) be defined by

$$
\sum_{k=0}^{\infty} b_{2 k} x^{2 k}=\frac{1}{2} \ln \frac{\sinh \frac{x}{2}}{\frac{x}{2}} .
$$

Let $\Omega \in \hat{\mathcal{B}}^{\prime}$ be the image of the element

$$
\exp \left(\sum_{k=1}^{\infty} b_{2 k} x_{2 k}\right) \in \mathbb{C}\left[\left[\left(x_{2 k}\right)_{k \in \mathbb{N}}\right]\right]
$$

under the morphism $\mathbb{C}\left[\left[\left(x_{2 k}\right)\right]\right] \rightarrow \hat{\mathcal{B}}^{\prime} \otimes_{\mathbb{Q}} \mathbb{C}$ of $\mathbb{C}$-algebras that maps $x_{2 k}$ to $w_{2 k}$.

For any $\mu \in \mathbb{C}$ we set $\Omega(\mu):=\sum_{k=0}^{\infty} \Omega_{k} \mu^{k}$, where $\Omega_{k}$ is the homogeneous component of degree $2 k$ of $\Omega$. (Note that $\Omega_{k}=0$ for odd $k$.)

The following remark is stated in [17].

Remark. The modified Bernoulli numbers are connected to the usual Bernoulli numbers $B_{1}, B_{2}, B_{3}, \ldots$ via

$$
b_{2 k}=\frac{B_{2 k}}{4 k(2 k) !}
$$

for all $k \in \mathbb{N}$. In addition to this, $b_{0}=0$.

The generating function of the (usual) Bernoulli numbers is given by

$$
\sum_{k=0}^{\infty} \frac{B_{k}}{k !} t^{k}=\frac{t}{e^{t}-1} .
$$

Note that $B_{k}=0$ for $k>1$ and $k$ odd. Furthermore, $B_{0}=1$ and $B_{1}=-\frac{1}{2}$.

Let $\Gamma$ be a Jacobi diagram and $u, u^{\prime}$ be two different univalent vertices of $\Gamma$. At least one of them should not belong to a component $\ell$ of $\Gamma$. Let $v$ (resp. $v^{\prime}$ ) be the vertex $u$ (resp. $u^{\prime}$ ) is attached to. The process of glueing the vertices $u$ and $u^{\prime}$ means to remove $u$ and $u^{\prime}$ together with the edges connecting them to $v$ resp. $v^{\prime}$ 
and to add a new edge between $v$ and $v^{\prime}$. Thus, we arrive at a new graph $\Gamma /\left(u, u^{\prime}\right)$, whose number of trivalent vertices is the numbers of trivalent vertices of $\Gamma$ and whose number of univalent vertices is the numbers of univalent vertices of $\Gamma$ minus two. To make it a Jacobi diagram we define the cyclic orientation of the flags at $v$ (resp. $\left.v^{\prime}\right)$ to be the cyclic orientation of the flags at $v$ (resp. $v^{\prime}$ ) in $\Gamma$ with the flag belonging to the edge connecting $v\left(\right.$ resp. $v^{\prime}$ ) with $u$ (resp. $u^{\prime}$ ) replaced by the flag belonging to the added edge.

For example, glueing the two univalent vertices of $w_{2}$ leads to the graph $\ominus$.

Of course, the process of glueing to univalent vertices given above does not work if $u$ and $u^{\prime}$ are the two univalent vertices of $\ell$, thus our assumption on $\Gamma$.

Definition 12. Let $\Gamma, \Gamma^{\prime}$ be two Jacobi diagrams, at least one of them without $\ell$ as a component and $U=\left\{u_{1}, \ldots, u_{n}\right\}$ resp. $U^{\prime}$ the sets of their univalent vertices. We define

$$
\hat{\Gamma}\left(\Gamma^{\prime}\right):=\sum_{\substack{f: U \leftrightarrow U^{\prime} \\ \text { injective }}}\left(\Gamma \cup \Gamma^{\prime}\right) /\left(u_{1}, f\left(u_{1}\right)\right) / \ldots /\left(u_{n}, f\left(u_{n}\right)\right),
$$

viewed as an element in $\hat{\mathcal{B}}$.

This induces for every $\gamma \in \hat{\mathcal{B}}$ a $\hat{\mathcal{B}}^{\mathrm{t}}$-linear map

$$
\hat{\gamma}: \hat{\mathcal{B}}^{\prime} \rightarrow \hat{\mathcal{B}}^{\prime}, \gamma^{\prime} \mapsto \hat{\gamma}\left(\gamma^{\prime}\right) .
$$

Example. Set $\partial:=\frac{1}{2} \hat{\ell}$. It is is an endomorphismus of $\hat{\mathcal{B}}^{\prime}$ of degree -2 . For example, $\partial-\sigma=\ominus$. By setting

$$
\partial\left(\gamma, \gamma^{\prime}\right):=\partial\left(\gamma \cup \gamma^{\prime}\right)-\partial(\gamma) \cup \gamma^{\prime}-\gamma \cup \partial\left(\gamma^{\prime}\right)
$$

for $\gamma, \gamma^{\prime} \in \hat{\mathcal{B}}^{\prime}$, we have the following formula for all $\gamma \in \hat{\mathcal{B}}^{\prime}$ :

$$
\partial\left(\gamma^{n}\right)=\left(\begin{array}{c}
n \\
1
\end{array}\right) \partial(\gamma) \gamma^{n-1}+\left(\begin{array}{c}
n \\
2
\end{array}\right) \partial(\gamma, \gamma) \gamma^{n-2}
$$

Acting by $\partial$ on a Jacobi diagram means to glue two of its univalent vertices in all possible ways, acting by $\partial(\cdot, \cdot)$ on two Jacobi diagrams means to connect them by glueing an univalent vertex of the first with an univalent vertex of the second in all possible ways. For example, we have

$$
\partial\left(w_{2 k}\right)=k \sum_{n=0}^{2 k-2} w_{n, 2 k-2-n}
$$

for $k \in \mathbb{N}$ and, by the IHX relation,

$$
\partial\left(w_{2 k_{1}}, w_{2 k_{2}}\right)=8 k_{1} k_{2} w_{2 k_{1}-1,2 k_{2}-1}
$$

for $k_{1}, k_{2} \in \mathbb{N}$.

Definition 13. Let $\Gamma, \Gamma^{\prime}$ be two Jacobi diagrams, at least one of them without $\ell$ as a component, and $U=\left\{u_{1}, \ldots, u_{n}\right\}$ resp. $U^{\prime}$ the sets of their univalent vertices. We define

$$
\left\langle\Gamma, \Gamma^{\prime}\right\rangle:=\sum_{\substack{f: U \rightarrow U^{\prime} \\ \text { bijective }}}\left(\Gamma \cup \Gamma^{\prime}\right) /\left(u_{1}, f\left(u_{1}\right)\right) / \ldots /\left(u_{n}, f\left(u_{n}\right)\right),
$$

viewed as an element in $\hat{\mathcal{B}}^{\mathrm{t}}$.

This induces a $\hat{\mathcal{B}}^{\mathrm{t}}$-bilinear map

$$
\langle\cdot, \cdot\rangle, \hat{\mathcal{B}}^{\prime} \times \hat{\mathcal{B}} \rightarrow \hat{\mathcal{B}}^{\mathrm{t}},
$$

which is symmetric on $\hat{\mathcal{B}}^{\prime} \times \hat{\mathcal{B}}^{\prime}$. 
Note that $\langle\Gamma, \Gamma\rangle$ is zero unless $\Gamma$ and $\Gamma^{\prime}$ have equal numbers of univalent vertices. In this case, the expression is the sum over all possibilities to glue the univalent vertices of $\Gamma$ with univalent vertices of $\Gamma^{\prime}$.

Proposition 2. The map $\langle 1, \cdot\rangle: \hat{\mathcal{B}} \rightarrow \hat{\mathcal{B}}^{\mathrm{t}}$ is the canonical projection map, i.e. it removes all non-trivalent components from a graph. Furthermore, for $\gamma \in \hat{\mathcal{B}}^{\prime}$ and $\gamma^{\prime} \in \hat{\mathcal{B}}$, we have

$$
\left\langle\gamma, \frac{\ell}{2} \gamma^{\prime}\right\rangle=\left\langle\partial \gamma, \gamma^{\prime}\right\rangle
$$

For $\gamma, \gamma^{\prime} \in \hat{\mathcal{B}}$, we have the following (combinatorial) formula:

$$
\left\langle\exp (\partial)\left(\gamma \gamma^{\prime}\right), 1\right\rangle=\left\langle\exp (\partial) \gamma, \exp (\partial) \gamma^{\prime}\right\rangle
$$

Proof. The formula (2.19) should be clear from the definitions.

Let us investigate (2.20) a bit more. We can assume that $\gamma$ and $\gamma^{\prime}$ are Jacobi diagrams with $l$ resp. $l^{\prime}$ univalent vertices and $l+l^{\prime}=2 n$ with $n \in \mathbb{N}_{0}$. So we have to prove

$$
\frac{\partial^{n}}{n !}\left(\gamma \gamma^{\prime}\right)=\sum_{\substack{m, m^{\prime}=0 \\ l-m=l^{\prime}-m^{\prime}}}^{\infty}\left\langle\frac{\partial^{m}}{m !} \gamma, \frac{\partial^{m^{\prime}}}{m^{\prime} !} \gamma^{\prime}\right\rangle,
$$

since $\langle\cdot, 1\rangle: \hat{\mathcal{B}} \rightarrow \hat{\mathcal{B}}^{\mathrm{t}}$ means to remove the components with at least one univalent vertex. Recalling the meaning of $\langle\cdot, \cdot\rangle$, it should be clear that (2.2) follows from the fact that applying $\frac{\partial^{k}}{k !}$ on a Jacobi diagram means to glue $k$ pairs of its univalent vertices in all possible ways.

2.3. $\Omega$ as an eigenvector of the operator $\partial$. As said above, the element $\Omega$ plays a central role in the "Wheeling Theorem" (see [17, where Bar-Natan, Le and Thurston have gave a knot theoretical prove of this theorem). Hitchin and Sawon ([10]) discovered that this theorem together with the ideas of Rozansky and Witten (15) can be used to deduce some interesting facts about characteristic classes on irreducible symplectic Kähler manifolds.

To prove our Hirzebruch-Riemann-Roch formula on irreducible symplectic Kähler manifolds along the ideas of Hitchin and Sawon, we could make use of the "Wheeling Theorem". However, it tells us a lot more about $\Omega$ than we actually need. In fact, we only need the statement given in theorem 2.1 about $\Omega$ to prove our results. Similarly, everything stated in 10 that is based on the "Wheeling Theorem" can also be based on theorem 2.1.

The following theorem is a corollary of lemma 6.2 in [17]. Nevertheless, we give another proof here, which does not use any knot theory.

Theorem 2.1. For each $\mu \in \mathbb{C}$, the graph $\Omega(\mu)$ is an eigenvector of the endomorphismus $\partial: \hat{\mathcal{B}}^{\prime} \rightarrow \hat{\mathcal{B}}^{\prime}$ with the eigenvalue $\frac{\mu^{2}}{48} \ominus$, i.e.

$$
\partial \Omega(\mu)=\frac{\mu^{2}}{48} \ominus \Omega(\mu)
$$

To make the proof of the theorem readable, we first state two lemmata. The first lemma is a combinatorial one, which makes use of the IHX relations. It collects some results of Chmutov, Dasbach and Duzhin Dasbach (see [5] and [6]).

Lemma 2.2. Let $\mathcal{W}$ be the subspace of $\hat{\mathcal{B}}$ that is spanned by all graphs $w_{i, j}$ with $i, j \in \mathbb{N}_{0}$. Let $P: \mathcal{W} \rightarrow \operatorname{Sym}_{\mathbb{Q}}^{3}\left(\left(x_{n}\right)_{n \in \mathbb{N}_{0}}\right)$ be the map defined by

$$
P\left(w_{i, j}\right)= \begin{cases}2 \sum_{l, m=0}^{\infty}(-1)^{l+m}\left(\begin{array}{c}
i \\
l
\end{array}\right)\left(\begin{array}{l}
j \\
m
\end{array}\right) x_{l} x_{m} x_{i+j-l-m} & \text { for } i+j \text { even } \\
0 & \text { for } i+j \text { odd } .\end{cases}
$$

Then $P$ is injective. 
Proof. First, we show that $P$ is well-defined.

For every $n \in \mathbb{N}_{0}$, let $\mathcal{W}_{n}$ be the subspace of $\mathcal{W}$ spanned by all $w_{i, j}$ with $i+j=n$. It is enough to show that for all $n \in \mathbb{N}_{0}$ there exists a injective map $P_{n}: \mathcal{W}_{n} \rightarrow$ $\operatorname{Sym}_{\mathbb{Q}}^{3}\left(\left(x_{n}\right)_{n \in \mathbb{N}_{0}}\right)$ that fulfills (2.22). Further, we can restrict ourselves to the case of even $n$ due to lemma 6.2 of [6], which says that $w_{i, j}$ is homologous to zero for odd $n$. (This follows at once from the anti-symmetry relation.)

For any $N>n$, the map $P_{\mathfrak{g r}(N)}$ defined in section 3.1 of $\left.\mid 5\right]$ can be taken as $P$ when restricted to $\mathcal{W}_{n}$. This is because of proposition 4.5 of $[5]$, where it is shown that $P_{\mathfrak{g l}(N)}$ evaluated at $w_{i, j}$ equals the right hand side of (2.22).

It remains to show that $P_{n}$ is injective. This can be proven by a dimension argument: By lemma 6.2 and lemma 6.8 of [6] the image of $\mathcal{W}_{n}$ under $P_{n}$ has at least the dimension of $\mathcal{W}_{n}$, so $P_{n}$ is injective.

Lemma 2.3. Let $B_{0}, B_{1}, B_{2}, \ldots$ denote the Bernoulli numbers. The following formula holds in $\operatorname{Sym}_{\mathbb{Q}}^{3}\left(\left(x_{n}\right)_{n \in \mathbb{N}_{0}}\right)$ :

$$
\begin{aligned}
& \sum_{k=2}^{\infty} \frac{B_{k}}{k !} \sum_{n=0}^{k-2} \sum_{l, m=0}^{\infty}(-1)^{l+m}\left(\begin{array}{c}
n \\
l
\end{array}\right)\left(\begin{array}{c}
k-2-n \\
m
\end{array}\right) x_{l} x_{m} x_{k-2-l-m} \\
& \quad+\sum_{i, j=2}^{\infty} \frac{B_{i}}{i !} \frac{B_{j}}{j !} \sum_{l, m=0}^{\infty}(-1)^{l+m}\left(\begin{array}{c}
i-1 \\
l
\end{array}\right)\left(\begin{array}{c}
j-1 \\
m
\end{array}\right) x_{l} x_{m} x_{i+j-2-l-m}=\frac{1}{12} x_{0}^{3} .
\end{aligned}
$$

Proof. In $\mathbb{Q}\left[X_{1}, X_{2}, X_{3}\right]$, we calculate

$$
\begin{aligned}
& \sum_{\pi \in \mathfrak{S}_{3}}\left(\sum_{k=2}^{\infty} \frac{B_{k}}{k !} \sum_{n=0}^{k-2} \sum_{l, m=0}^{\infty}(-1)^{l+m}\left(\begin{array}{c}
n \\
l
\end{array}\right)\left(\begin{array}{c}
k-2-n \\
m
\end{array}\right) X_{\pi(1)}^{l} X_{\pi(2)}^{m} X_{\pi(3)}^{k-2-l-m}\right. \\
& \left.+\sum_{i, j=2}^{\infty} \frac{B_{i}}{i !} \frac{B_{j}}{j !} \sum_{l, m=0}^{\infty}(-1)^{l+m}\left(\begin{array}{c}
i-1 \\
l
\end{array}\right)\left(\begin{array}{c}
j-1 \\
m
\end{array}\right) X_{\pi(1)}^{l} X_{\pi(2)}^{m} X_{\pi(3)}^{i+j-2-l-m}\right) \\
& =\sum_{\pi \in \mathfrak{S}_{3}}\left(\sum_{k=2}^{\infty} \frac{B_{k}}{k !} \sum_{n=0}^{k-2}\left(X_{\pi(3)}-X_{\pi(1)}\right)^{n}\left(X_{\pi(3)}-X_{\pi(2)}\right)^{k-2-n}\right. \\
& \left.+\sum_{i, j=2}^{\infty} \frac{B_{i}}{i !} \frac{B_{j}}{j !}\left(X_{\pi(3)}-X_{\pi(1)}\right)^{i-1}\left(X_{\pi(3)}-X_{\pi(2)}\right)^{j-1}\right) \\
& =\sum_{\pi \in \mathfrak{S}_{3}}\left(\frac{1}{X_{\pi(1)}-X_{\pi(2)}}\left(\begin{array}{c}
\frac{1}{X_{\pi(3)}-X_{\pi(2)}} \sum_{k=2}^{\infty} \frac{B_{k}}{k !}\left(X_{\pi(3)}-X_{\pi(2)}\right)^{k} \\
-\frac{1}{X_{\pi(3)}-X_{\pi(1)}} \sum_{k=2}^{\infty} \frac{B_{k}}{k !}\left(X_{\pi(3)}-X_{\pi(1)}\right)^{k}
\end{array}\right)\right. \\
& +\frac{1}{\left(X_{\pi(3)}-X_{\pi(1)}\right)\left(X_{\pi(3)}-X_{\pi(2)}\right)} \\
& \left.\cdot\left(\sum_{k=2}^{\infty} \frac{B_{k}}{k !}\left(X_{\pi(3)}-X_{\pi(1)}\right)^{k}\right)\left(\sum_{k=2}^{\infty} \frac{B_{k}}{k !}\left(X_{\pi(3)}-X_{\pi(2)}\right)^{k}\right)\right) \\
& =\sum_{\pi \in \mathfrak{S}_{3}}\left(\frac{1}{X_{\pi(1)}-X_{\pi(2)}}\left(\begin{array}{c}
\frac{1}{\exp \left(X_{\pi(3)}-X_{\pi(2)}\right)-1}-\frac{1}{X_{\pi(3)}-X_{\pi(2)}} \\
-\frac{1}{\exp \left(X_{\pi(3)}-X_{\pi(1)}\right)-1}+\frac{1}{X_{\pi(3)}-X_{\pi(1)}}
\end{array}\right)\right. \\
& +\left(\frac{1}{\exp \left(X_{\pi(3)}-X_{\pi(1)}\right)-1}-\frac{1}{X_{\pi(3)}-X_{\pi(1)}}+\frac{1}{2}\right)
\end{aligned}
$$




$$
\left.\cdot\left(\frac{1}{\exp \left(X_{\pi(3)}-X_{\pi(2)}\right)-1}-\frac{1}{X_{\pi(3)}-X_{\pi(2)}}+\frac{1}{2}\right)\right)=\frac{1}{2} .
$$

This proves the lemma because there is a well-defined $\mathbb{Q}$-linear map

$$
\operatorname{Sym}_{\mathbb{Q}}^{3}\left(\left(x_{n}\right)_{n \in \mathbb{N}_{0}}\right) \rightarrow \mathbb{Q}\left[X_{1}, X_{2}, X_{3}\right], x_{i} x_{j} x_{k} \mapsto \sum_{\pi \in \mathfrak{S}_{3}} X_{\pi(i)}^{i} X_{\pi(2)}^{j} X_{\pi(3)}^{k},
$$

which is injective.

Proof of the theorem. Since $\partial$ is a linear operator of degree -2 on $\hat{\mathcal{B}}^{\prime}$ and $\Omega(\mu)=$ $\sum_{k=0}^{\infty} \Omega_{2 k} \mu^{2 k}$, we can assume that $\mu=1$. Set $\Gamma:=\sum_{k=1}^{\infty} b_{2 k} w_{2 k}$. We have

$$
\begin{aligned}
\partial \Omega & =\partial \exp (\Gamma)=\sum_{n=0}^{\infty} \frac{\partial\left(\Gamma^{n}\right)}{n !} \\
& =\sum_{n=1}^{\infty} \partial(\Gamma) \frac{\Gamma^{n-1}}{(n-1) !}+\sum_{n=2}^{\infty} \frac{\partial(\Gamma, \Gamma)}{2} \frac{\Gamma^{n-2}}{(n-2) !} \\
& =\left(\partial \Gamma+\frac{1}{2} \partial(\Gamma, \Gamma)\right) \Omega,
\end{aligned}
$$

so we see that $\Omega$ is an eigenvector. We just have to calculate the eigenvalue:

$$
\begin{aligned}
\partial \Gamma+\frac{1}{2} \partial(\Gamma, \Gamma) & =\sum_{k=1}^{\infty} b_{2 k} \partial w_{2 k}+\sum_{i, j=1}^{\infty} b_{2 i} b_{2 j} \frac{\partial\left(w_{2 i}, w_{2 j}\right)}{2} \\
& =\sum_{k=1}^{\infty} k b_{2 k} \sum_{n=0}^{2 k-2} w_{n, 2 k-2-n}+\sum_{i, j=1}^{\infty} 4 i j b_{2 i} b_{2 j} w_{2 i-1,2 j-1} \\
& =\frac{1}{4} \sum_{k=1}^{\infty} \frac{B_{2 k}}{(2 k) !} \sum_{n=0}^{2 k-2} w_{n, 2 k-2-n}+\frac{1}{4} \sum_{i, j=1}^{\infty} \frac{B_{2 i}}{(2 i) !} \frac{B_{2 j}}{(2 j) !} w_{2 i-1,2 j-1} \\
& =\frac{1}{4} \sum_{k=2}^{\infty} \frac{B_{k}}{k !} \sum_{n=0}^{k-2} w_{n, k-2-n}+\frac{1}{4} \sum_{i, j=2}^{\infty} \frac{B_{i}}{i !} \frac{B_{j}}{j !} w_{i-1, j-1} .
\end{aligned}
$$

Applying the (injectiv) map $P$ of lemma 2.2 and using lemma 2.3 yields

$$
\begin{array}{r}
P\left(\partial \Gamma+\frac{1}{2} \partial(\Gamma, \Gamma)\right)=\frac{1}{4} P\left(\sum_{k=2}^{\infty} \frac{B_{k}}{k !} \sum_{n=0}^{k-2} w_{n, k-2-n}+\sum_{i, j=2}^{\infty} \frac{B_{i}}{i !} \frac{B_{j}}{j !} w_{i-1, j-1}\right) \\
=\frac{1}{24} x_{0}^{3}=P\left(\frac{1}{48} w_{0,0}\right)=P\left(\frac{1}{48} \ominus\right) .
\end{array}
$$

Because of the injectivity of $P$, this proves the theorem.

\section{Rozansky-Witten CLASSES}

The idea to associate to every graph $\Gamma$ and every hyperkähler manifold $X$ a cohomology class $\mathrm{RW}_{X}(\Gamma)$ is due to Rozansky and Witten (c.f. 15]). Kapranov showed in 12] that the metric structure of a hyperkähler manifold is not nessessary to define these classes. It was his idea to build the whole theory upon the Atiyah class and the symplectic structure of a symplectic Kähler manifold. We will use his definition of Rozansky-Witten classes in this section. 
3.1. Rozansky-Witten classes in general. Let $k$ be a field of characteristic zero, $V$ a finite-dimensional $k$-vector space, $A=\bigoplus_{i=0}^{\infty} A_{i}$ a $\mathbb{Z}$-graded (supercommutative $k$-algebra and $\sigma$ a symplectic form on $V$.

We shall use this general setting later in the case that $V=\mathcal{T}_{X, x}$ is the holomorphic tangent space of a complex manifold $X$ at a point $x$ and $A=\bar{\Omega}_{X, x}^{*}$ is the Graßmann algebra of anti-holomorphic forms at $x$.

For every Jacobi diagram $\Gamma$ with $k$ trivalent and $l$ univalent vertices and every $\alpha \in \mathrm{Sym}^{3} V \otimes_{k} A_{1}$, we define an element

$$
\mathrm{RW}_{\sigma, \alpha}(\Gamma) \in \bigwedge^{l}\left(V^{*}\right) \otimes A_{k}
$$

by the following procedure:

Let $T$ denote the set of trivalent vertices, $U$ the set of univalent vertices, $E$ the set of edges and $F$ the set of flags of $\Gamma$. So, $l=|U|$ and $k=|T|$. The Jacobi diagram $\Gamma$ defines the map

$$
\begin{aligned}
\Phi^{\Gamma}:\left(\mathrm{Sym}^{3} V \otimes A_{1}\right)^{\otimes T} \otimes(\operatorname{End} V)^{\otimes U} & \stackrel{(1)}{\longrightarrow}\left(\bigotimes_{t \in T} V^{\otimes t}\right) \otimes A_{1}^{\otimes T} \otimes V^{\otimes U} \otimes\left(V^{*}\right)^{\otimes U} \\
& \stackrel{(2)}{\longrightarrow} V^{\otimes F} \otimes\left(V^{*}\right)^{\otimes U} \otimes A_{1}^{\otimes T} \\
& \stackrel{(3)}{\longrightarrow} \bigotimes_{e \in E} V^{\otimes e} \otimes\left(V^{*}\right)^{\otimes U} \otimes A_{1}^{\otimes T} \\
& \stackrel{(4)}{\longrightarrow}\left(V^{\otimes 2}\right)^{\otimes \frac{3 k+l}{2}} \otimes\left(V^{*}\right)^{\otimes l} \otimes\left(A_{1}\right)^{\otimes k} \\
& \stackrel{(5)}{\longrightarrow} \bigwedge^{l}\left(V^{*}\right) \otimes A_{k},
\end{aligned}
$$

where

1. is induced by the inclusions of the symmetric tensors $\operatorname{Sym}^{3} V$ in the spaces $V^{\otimes t}$ with $t \in T$ (note again that $t$ is a set of three elements) and the canonical identification End $V=V \otimes V^{*}$,

2. is induced by the canonical bijection $U \amalg \coprod_{t \in T} t \rightarrow F$ which is on each $t \in T$ the inclusion of the subset $t$ in $F$ and which maps each $u \in U$ to the flag belonging to $u$,

3. is induced by the associativity of the tensor product (note that $\coprod_{e \in E} e=F$ ),

4. is induced by choosing total orderings of the set $E$, the sets $e \in E$, the set $U$ and the set $T$, which are compatible with the orientation of the graph $\Gamma$, and

5 . is given by $\left(\left(v_{1} \otimes v_{2}\right) \otimes \cdots \otimes\left(v_{3 k+l-1} \otimes v_{3 k+l}\right)\right) \otimes\left(\alpha_{1} \otimes \cdots \otimes \alpha_{l}\right) \otimes\left(a_{1} \otimes \cdots \otimes a_{k}\right)$ $\mapsto \sigma\left(v_{1}, v_{2}\right) \cdots \sigma\left(v_{3 k+l-1}, v_{3 k+l}\right) \cdot\left(\alpha_{1} \wedge \cdots \wedge \alpha_{l}\right) \otimes\left(a_{1} \cdots a_{k}\right)$.

The map $\Phi^{\Gamma}$ is independent of the specific choice made in (4). One defines

$$
\mathrm{RW}_{\sigma, \alpha}(\Gamma):=\Phi^{\Gamma}\left(\alpha^{\otimes T} \otimes\left(\mathrm{id}_{V}\right)^{\otimes U}\right) .
$$

The following proposition summarizes some of the properties of the maps $\Phi^{\Gamma}$ we need later on. All of them follow directly from the definitions.

Proposition 3. Identifying the set of the univalent vertices of $\ell$ with the set $\{1,2\}$, we have

$$
\Phi^{\ell}:(\operatorname{End} V)^{\otimes 2} \rightarrow \bigwedge^{2}\left(V^{*}\right),\left(v_{1} \otimes \lambda_{1}\right) \otimes\left(v_{2} \otimes \lambda_{2}\right) \mapsto \sigma\left(v_{1}, v_{2}\right) \cdot\left(\lambda_{1} \wedge \lambda_{2}\right)
$$

for all $v_{1}, v_{2} \in V, \lambda_{1}, \lambda_{2} \in V^{*}$.

Let $k \in \mathbb{N}_{0}$. Identifying both the set of the univalent vertices and the set of the trivalent vertices of the wheel $w_{2 k}$ (viewed as a Jacobi diagram) with the set 
$\{1, \ldots, 2 k\}$, we have

$$
\begin{aligned}
& 3.5) \quad \Phi^{w_{2 k}}:\left(\operatorname{Sym}^{3} V \otimes A_{1}\right)^{\otimes 2 k} \otimes(\operatorname{End} V)^{\otimes 2 k} \rightarrow \bigwedge^{2 k}\left(V^{*}\right) \otimes A_{2 k} \\
& \bigotimes_{i=1}^{2 k}\left(v_{i}^{3} \otimes a_{i}\right) \otimes \bigotimes_{i=1}^{2 k}\left(w_{i} \otimes \lambda_{i}\right) \mapsto-\prod_{i \in \mathbb{Z} /(2 k)}\left(\sigma\left(v_{i}, v_{i+1}\right) \cdot \sigma\left(v_{i}, w_{i}\right)\right) \cdot \bigwedge_{i=1}^{2 k} \lambda_{i} \otimes \prod_{i=1}^{2 k} a_{i}
\end{aligned}
$$

for all $v_{i}, w_{i} \in V, \lambda_{i} \in V^{*}$ and $a_{i} \in A_{1}$.

Let $\Gamma$ and $\Gamma^{\prime}$ be two Jacobi diagrams with univalent vertices $U$ and $U^{\prime}$ and trivalent vertices $T$ and $T^{\prime}$. Let the cardinalities of $T, T^{\prime}, U, U^{\prime}$ be $k, k^{\prime}, l, l^{\prime}$. Then the diagram

$$
\begin{aligned}
& \left(\left(\mathrm{Sym}^{3} V \otimes A_{1}\right)^{\otimes T} \otimes(\operatorname{End} V)^{\otimes U}\right) \\
& \left(\bigwedge^{2 l}\left(V^{*}\right) \otimes A_{2 k}\right) \\
& \otimes\left(\left(\mathrm{Sym}^{3} V \otimes A_{1}\right)^{\otimes T^{\prime}} \otimes(\mathrm{End} V)^{\otimes U^{\prime}}\right) \stackrel{\Phi^{\Gamma} \otimes \Phi^{\Gamma^{\prime}}}{\longrightarrow} \quad \otimes\left(\bigwedge^{2 l^{\prime}}\left(V^{*}\right) \otimes A_{2 k^{\prime}}\right)
\end{aligned}
$$

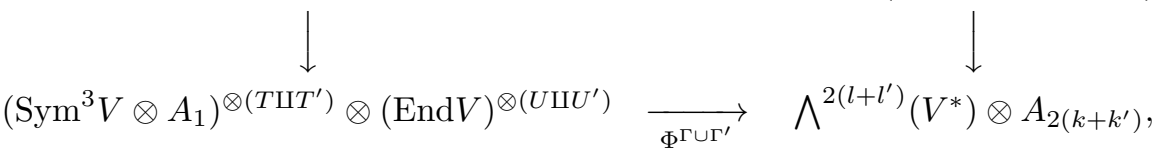

where the vertical maps are the canonical ones, and the diagram

$$
\begin{aligned}
& \left(\left(\operatorname{Sym}^{3} V \otimes A_{1}\right)^{\otimes T} \otimes(\operatorname{End} V)^{\otimes U}\right) \quad \stackrel{\Phi^{\Gamma} \otimes \Phi^{\Gamma^{\prime}}}{\longrightarrow}\left(\bigwedge^{2 l}\left(V^{*}\right) \otimes A_{2 k}\right) \\
& \otimes\left(\left(\operatorname{Sym}^{3} V \otimes A_{1}\right)^{\otimes T^{\prime}} \otimes(\operatorname{End} V)^{\otimes U^{\prime}}\right) \stackrel{\longrightarrow}{\longrightarrow} \otimes\left(\bigwedge^{2 l^{\prime}}\left(V^{*}\right) \otimes A_{2 k^{\prime}}\right) \\
& \left(\mathrm{Sym}^{3} V \otimes A_{1}\right)^{\otimes T} \otimes\left(\mathrm{Sym}^{3} V \otimes A_{1}\right)^{\otimes T^{\prime}} \underset{\Phi^{\left\langle\Gamma, \Gamma^{\prime}\right\rangle}}{ } \quad \begin{array}{l}
\downarrow \\
A_{2\left(k+k^{\prime}\right)},
\end{array}
\end{aligned}
$$

where the left vertical map is induced by tensoring with the identity on $V$ and the right one is just (1.4), commute.

3.2. Rozansky-Witten invariants of symplectic Kähler manifolds. All our Kähler manifolds are assumed to be compact.

Let $X$ be a symplectic Kähler manifold, $\sigma$ a fixed symplectic form and $\alpha$ a Dolbeault representative of the Atiyah class of $X$. We can use $\sigma$ to identify the tangent bundle $\mathcal{T}_{X}$ of $X$ with the cotangent bundle $\Omega_{X}$. Doing this, $\alpha$ can be viewed as an element of $A^{1}\left(X, \mathcal{T}_{X}^{\otimes 3}\right)$. Here, $A^{k}(X, E)$ denotes the space of $(0, k)$-forms with values in a holomorphic vector bundle $E$. We set $A^{l, k}(X):=A^{k}\left(X, \Omega_{X}^{l}\right)$.

The following proposition was proven by Kapranov in [12].

Proposition 4. The values of $\alpha$ are symmetric tensors, i.e. $\alpha \in A^{1}\left(X, \operatorname{Sym}^{3} \mathcal{T}_{X}\right)$.

Definition 14. For every Jacobi diagram $\Gamma$ with $k$ trivalent and $l$ univalent vertices, one defines

$$
\operatorname{RW}_{\sigma}(\Gamma) \in \mathrm{H}^{k}\left(X, \Omega^{l}\right)
$$

to be the Dolbeault cohomology class of the $(\bar{\partial}-)$ closed $(l, k)$-form

$$
\left(x \mapsto \mathrm{RW}_{\sigma_{x}, \alpha_{x}}(\Gamma)\right) \in A^{l, k}(X) .
$$

(That the form defined in (3.9) is $\bar{\partial}$-closed follows from the fact that $\sigma$ and $\alpha$ are $\bar{\partial}$-closed.) 
For a $\mathbb{Q}$-linear combination $\gamma$ of Jacobi diagrams, $\mathrm{RW}_{\sigma}(\gamma)$ is defined by linear extension.

In 12 , Kapranov also showed the following proposition, which is crucial for the next definition.

Proposition 5. If $\gamma$ is a $\mathbb{Q}$-linear combination of Jacobi diagrams that is zero modulo the anti-symmetry and IHX relations, then $\mathrm{RW}_{\sigma}(\gamma)=0$.

Definition 15. We define a linear map

$$
\mathrm{RW}_{\sigma}: \hat{\mathcal{B}} \rightarrow \mathrm{H}^{*}\left(X, \Omega^{*}\right)
$$

which maps $\hat{\mathcal{B}}_{k, l}$ into $\mathrm{H}^{k}\left(X, \Omega^{l}\right)$, by mapping a homology class of a Jacobi diagram $\Gamma$ to $\mathrm{RW}_{\sigma}(\Gamma)$.

The values of the just defined map $\mathrm{RW}_{\sigma}$ are called Rozansky-Witten classes of the symplectic Kähler manifold $X$.

3.3. Examples of Rozansky-Witten classes. Let $X$ and $\sigma$ be as before.

Example. The Dolbeault cohomology class $[\sigma] \in \mathrm{H}^{2,0}(X)$ is a Rozansky-Witten class, more precisely, we have

$$
\mathrm{RW}_{\sigma}(\ell)=2 \sigma
$$

which follows from (3.4).

The following example is due to Hitchin and Sawon [10]. It is of great importance for their and our results.

Example. Let $\operatorname{ch}(X)=\sum_{k=0}^{\infty} s_{2 k} /(2 k) !, s_{2 k} \in \mathrm{H}^{2 k, 2 k}(X)$, be the Chern character of $X$. Then

$$
\operatorname{RW}_{\sigma}\left(w_{2 k}\right)=-s_{2 k}
$$

for all $k \in \mathbb{N}_{0}$. (Note that for a symplectic manifold $\operatorname{ch}(X)$ has no term in degree $(k, k)$ for $k$ odd.)

Since the algebra of characteristic classes of $X$ is spanned by the classes $s_{2 k}$, every characteristic class is a Rozansky-Witten class due to proposition 6 below.

A proof of (3.12) is given by Hitchin and Sawon in [10], where the RozanskyWitten invariants are defined by using the Riemann curvature tensor of a hyperkähler metric of $X$ instead of the Atiyah class. An idea of the proof in our context is given below.

Idea of the proof of (3.12). As remarked in 12] by Kapranov, a Dolbeault representative of the Atiyah class $\alpha \in A^{1}\left(X, \Omega_{X} \otimes \operatorname{End} \mathcal{T}_{X}\right)$ on every Kähler manifold $X$ is related to the characteristic classes $s_{k} \in \mathrm{H}^{k, k}(X)$ of $X$ in the following way: A Dolbeault representative of $s_{k}$ is given by $\operatorname{Alt}\left(\operatorname{tr}\left(\alpha^{k}\right)\right)$. Here, $\alpha^{k} \in A^{k}\left(X, \Omega_{X}^{\otimes k} \otimes \operatorname{End} \mathcal{T}_{X}\right)$ means taking the $k$-th product of $\alpha$ viewed as $(0,1)$-form, giving an element of $A^{k}\left(X, \Omega_{X}^{\otimes k} \otimes_{X}\left(\operatorname{End} \mathcal{T}_{X}\right)^{\otimes k}\right)$, and then using the associative algebra structure of End $\mathcal{T}_{X}$. Further, tr means taking the trace on $\operatorname{End} \mathcal{T}_{X}$, and Alt is induced by the canonical projection $\Omega_{X}^{\otimes k} \rightarrow \Omega_{X}^{k}$.

That this procedure on symplectic Kähler manifolds is essentially the same as taking the Rozansky-Witten class of a wheel follows from (3.5).

Example. The Todd genus of a symplectic complex manifold $X$ is given by

$$
\operatorname{td}(X)=\exp \left(-2 \sum_{k=0}^{\infty} b_{2 k} s_{2 k}\right)
$$


with $b_{2 k}$ being a modified Bernoulli number (see 10] for this statement). Thus,

$$
\operatorname{td}(X)=\operatorname{RW}_{\sigma}\left(\Omega^{2}\right) .
$$

\subsection{Properties of Rozansky-Witten classes.}

Proposition 6. The map $\mathrm{RW}_{\sigma}: \hat{\mathcal{B}} \rightarrow \mathrm{H}^{*, *}(X)$ is a morphism of graded $\mathbb{Q}$ algebras.

Furthermore, we have

$$
\operatorname{RW}_{\sigma}\left(\left\langle\Gamma, \Gamma^{\prime}\right\rangle\right)=\left\langle\operatorname{RW}_{\sigma}(\Gamma), \operatorname{RW}_{\sigma}\left(\Gamma^{\prime}\right)\right\rangle
$$

for all $\Gamma, \Gamma^{\prime} \in \hat{\mathcal{B}}$.

Proof. The statements follow from (3.6) and (3.7).

The following proposition is also stated in [10] in a slightly different notation. With the formalism introduced here, we can give a compact proof.

Proposition 7. If $X$ is irreducible, we have

$$
\operatorname{RW}_{\sigma}(\ominus)=\frac{2 \int_{X} c_{2}(X) \exp (\sigma+\bar{\sigma})}{n \int \exp _{X}(\sigma+\bar{\sigma})} \cdot[\bar{\sigma}] .
$$

Proof. Due to the irreducibility of $X$, i.e. $\mathrm{H}^{2 k}\left(X, \mathcal{O}_{X}\right)=\mathbb{C} \cdot[\bar{\sigma}]$ for all $k \in \mathbb{N}_{0}$, we can write

$$
\alpha=\frac{\int_{X} \alpha \exp (\sigma+\bar{\sigma})}{n \int_{X} \exp (\sigma+\bar{\sigma})} \cdot[\bar{\sigma}]
$$

for all $\alpha \in \mathrm{H}^{0,2}(X)$. Using this, we have

$$
\begin{aligned}
& \mathrm{RW}_{\sigma}(\ominus)=\mathrm{RW}_{\sigma}\left(\frac{1}{2}\left\langle w_{2}, \ell\right\rangle\right)=\frac{1}{2}\left\langle\mathrm{RW}_{\sigma}\left(w_{2}\right), \mathrm{RW}_{\sigma}(\ell)\right\rangle \\
& \quad=\left\langle-s_{2}, \sigma\right\rangle=2\left\langle c_{2}(X), \exp \sigma\right\rangle=\frac{2 \int_{X}\left\langle c_{2}(X), \exp \sigma\right\rangle \exp (\sigma+\bar{\sigma})}{n \int_{X} \exp (\sigma+\bar{\sigma})} \cdot[\bar{\sigma}],
\end{aligned}
$$

which proves the proposition because of proposition 1 .

\section{The Euler Characteristic of a line Bundle in terms of the QUADRATIC FORM}

Let $X$ be an irreducible symplectic Kähler manifold with symplectic form $\sigma$.

4.1. The quadratic form on an irreducible symplectic manifold. Let us assume $\int_{X}(\sigma \bar{\sigma})^{n}=1$ for this subsection.

Definition 16. We set

$$
\begin{aligned}
f_{X}: \mathrm{H}^{2}(X, \mathbb{C}) \rightarrow \mathbb{C} \\
\quad \alpha \mapsto \frac{n}{2} \int_{X} \alpha^{2}(\sigma \bar{\sigma})^{n-1}+(1-n)\left(\int_{X} \alpha \bar{\sigma}(\sigma \bar{\sigma})^{n-1}\right)\left(\int_{X} \alpha \sigma(\sigma \bar{\sigma})^{n-1}\right) .
\end{aligned}
$$

Remark. The form $f_{X}$ is a multiple of the Beauville-Bogomolov quadratic form $q_{X}$ on $X$. A detailed discussion of $f_{X}$ and $q_{X}$ can be found in [11]. Note that $f_{X}(\alpha)=\frac{n}{2} \int \alpha^{2}(\sigma \bar{\sigma})^{n-1}$ if $\alpha \in \mathrm{H}^{1,1}(X)$.

We need a fact about $f_{X}$, which was proved by Huybrechts in [11. 
Proposition 8. Assume that $\alpha \in \mathrm{H}^{4 j}(X, \mathbb{C})$ is a characteristic class of $X$ (it suffices to assume that $\alpha$ is of type $(2 j, 2 j)$ on all small deformations of $X)$. Then there is a constant $c_{\alpha} \in \mathbb{C}$, depending on $\alpha$, with

$$
\int_{X} \alpha \beta^{2(n-j)}=c_{\alpha} \cdot\left(f_{X}(\beta)\right)^{n-j}
$$

for all $\beta \in \mathrm{H}^{2}(X, \mathbb{C})$.

Corollary 4.1. For all $\beta \in \mathrm{H}^{2}(X, \mathbb{C})$, we have

$$
\int_{X} \beta^{2 n}=\left(\begin{array}{c}
2 n \\
n
\end{array}\right)\left(f_{X}(\beta)\right)^{n}
$$

and

$$
\int_{X} c_{2}(X) \beta^{2 n-2}=\left(\begin{array}{c}
2 n-2 \\
n-1
\end{array}\right)\left(\int_{X} c_{2}(X)(\sigma \bar{\sigma})^{n-1}\right) \cdot\left(f_{X}(\beta)\right)^{n-1} .
$$

Proof. Set $\alpha=1$ in (4.2). To obtain $c_{1}$, compute both sides with $\beta=\sigma+\bar{\sigma}$, which proves the first equation.

Then set $\alpha=c_{2}(X)$ in (4.2). To obtain $c_{c_{2}(X)}$ compute both sides with $\beta=\sigma+\bar{\sigma}$, which leads to the second equation.

\subsection{A Hirzebruch-Riemann-Roch formula. Let us define the number}

$$
\lambda:=\frac{24 n \int_{X} \exp (\sigma+\bar{\sigma})}{\int_{X} c_{2}(X) \exp (\sigma+\bar{\sigma})}
$$

for the pair $(X, \sigma)$. It is well-defined (i.e. the denominator does not vanish) because $\int_{X} c_{2}(X) \exp (\sigma+\bar{\sigma})$ equals the $\mathrm{L}^{2}$-norm of the Riemann curvature tensor of $X$ (having been equipped with a hyperkähler metric compatible with the given symplectic structure) up to a positive constant (see [10]). But if the Riemann curvature tensor vanishes, $X$ is a torus, which contradicts the assumption on irreducibility.

We continue to write $b_{2 k}$ for the modified Bernoulli numbers and $s_{2 k}$ for the homogeneous components of $\operatorname{ch}(X)$ (up to the constant $(2 k) !$ ).

Proposition 9. For all $\mu \in \mathbb{C}$ with $\mu^{2}+\mu^{-2}=2+\lambda$, we have

$$
\int_{X} \operatorname{td}(X) \exp (\sigma+\bar{\sigma})=\int_{X} \exp \left(-\sum_{k=0}^{\infty} b_{2 k} s_{2 k} \cdot\left(\mu^{2 k}+\mu^{-2 k}\right)\right) .
$$

Proof. Note that $\langle\Omega, \Omega\rangle=\left(\Omega(\mu), \Omega\left(\mu^{-1}\right)\right\rangle$. Then

$$
\begin{aligned}
& \int_{X} \operatorname{td}(X) \exp (\sigma+\bar{\sigma})=\int_{X} \operatorname{RW}_{\sigma}\left(\Omega^{2}\right) \exp (\sigma+\bar{\sigma}) \\
& =\int_{X}\left\langle\operatorname{RW}_{\sigma}\left(\Omega^{2}\right), \exp \sigma\right\rangle \exp (\sigma+\bar{\sigma})=\int_{X} \operatorname{RW}_{\sigma}\left(\left\langle\Omega^{2}, \exp (\ell / 2)\right\rangle\right) \exp (\sigma+\bar{\sigma}) \\
& =\int_{X} \operatorname{RW}_{\sigma}\left(\left\langle\exp (\partial)\left(\Omega^{2}\right), 1\right\rangle\right) \exp (\sigma+\bar{\sigma}) \\
& =\int_{X} \operatorname{RW}_{\sigma}(\langle\exp (\partial) \Omega, \exp (\partial) \Omega\rangle) \exp (\sigma+\bar{\sigma}) \\
& =\int_{X} \operatorname{RW}_{\sigma}(\langle\exp (\partial) \Omega, \exp (\partial) \Omega\rangle) \mathrm{RW}_{\sigma}\left(\exp \left(\frac{\lambda}{48} \ominus\right)\right) \exp (\sigma) \\
& =\int_{X} \mathrm{RW}_{\sigma}\left(\exp \left(\frac{2+\lambda}{48} \ominus\right)\langle\Omega, \Omega\rangle\right) \exp (\sigma) \\
& =\int_{X} \mathrm{RW}_{\sigma}\left(\exp \left(\frac{2+\lambda}{48} \ominus\right)\left\langle\Omega(\mu), \Omega\left(\mu^{-1}\right)\right\rangle\right) \exp (\sigma)
\end{aligned}
$$




$$
\begin{aligned}
& =\int_{X} \operatorname{RW}_{\sigma}\left(\left\langle\exp (\partial) \Omega(\mu), \exp (\partial) \Omega\left(\mu^{-1}\right)\right\rangle\right) \exp (\sigma) \\
& =\int_{X} \operatorname{RW}_{\sigma}\left(\left\langle\exp (\partial)\left(\Omega(\mu) \Omega\left(\mu^{-1}\right)\right), 1\right\rangle\right) \exp (\sigma) \\
& =\int_{X} \operatorname{RW}_{\sigma}\left(\left\langle\Omega(\mu) \Omega\left(\mu^{-1}\right), \exp (\ell / 2)\right\rangle\right) \exp (\sigma) \\
& =\int_{X}\left\langle\mathrm{RW}_{\sigma}\left(\Omega(\mu) \Omega\left(\mu^{-1}\right)\right), \exp (\sigma)\right\rangle \exp (\sigma)=\int_{X} \mathrm{RW}_{\sigma}\left(\Omega(\mu) \Omega\left(\mu^{-1}\right)\right) \exp (\sigma) \\
& =\int_{X} \operatorname{RW}_{\sigma}\left(\Omega(\mu) \Omega\left(\mu^{-1}\right)\right)=\int_{X} \exp \left(-\sum_{k=0}^{\infty} b_{2 k} s_{2 k} \cdot\left(\mu^{2 k}+\mu^{-2 k}\right)\right)
\end{aligned}
$$

Remark. The right hand side of (4.6) is polynomial in $\lambda$ since

$$
\mu^{n}+\mu^{-n}=2 T_{n}\left( \pm \sqrt{\frac{z}{4}+\frac{1}{2}}\right)
$$

for all $\mu, z \in \mathbb{C}$ with $\mu^{2}+\mu^{-2}=z$ and $n \in \mathbb{N}_{0}$. Here, $T_{n}$ denotes the $n^{\text {th }}$ Chebychev polynomial. Note that $T_{n}$ is even for $n$ even.

Proof. We can assume that $z \in[-2,2]$. If follows that $\mu= \pm e^{ \pm i x}$ with $x=$ $\frac{1}{2} \arccos \left(\frac{z}{2}\right)=\arccos \left(\sqrt{\frac{z}{4}+\frac{1}{2}}\right)$. By definition of the Chebychev polynomials, we have

$$
\mu^{n}+\mu^{-n}=2 \cos \left(n \cdot \arccos \left( \pm \sqrt{\frac{z}{4}+\frac{1}{2}}\right)\right)=2 T_{n}\left( \pm \sqrt{\frac{z}{4}+\frac{1}{2}}\right) .
$$

Definition 17. For every line bundle $L$ on $X$, we call the rational number

$$
\lambda(L):= \begin{cases}\frac{24 n \int_{X} \operatorname{ch}(L)}{\int c_{2}(X) \operatorname{ch}(L)} & \text { if well-defined } \\ 0 & \text { otherwise }\end{cases}
$$

the characteristic value of $L$.

We could have defined a characteristic value $\lambda(\alpha)$ for every cohomology class $\alpha \in \mathrm{H}^{2}(X, \mathbb{C})$ so that $\lambda(L)=\lambda\left(c_{1}(L)\right)$. Then the number $\lambda$ for the pair $(X, \sigma)$ defined above is just $\lambda(\sigma+\bar{\sigma})$.

Remark. If $\lambda(L)=0$, then

$$
\chi(L)=\chi\left(\mathcal{O}_{X}\right)=\int_{X} \operatorname{td}(X) .
$$

Proof. If $\lambda(X)=0$, then $\int_{X} \operatorname{ch}(L)=0$ or $\int_{X} c_{2}(X) \operatorname{ch}(L)=0$. In both cases, $f_{X}\left(c_{1}(L)\right)=0$ due to the corollaries 4.1 and 4.1. Because of the proposition 8 and the usual Hirzebruch-Riemann-Roch formula, the assertion follows.

Proposition 10. For the characteristic value of line bundle $L$ on $X$,

$$
\lambda(L)=\frac{12 \int_{X} c_{1}(L)^{2}(\sigma \bar{\sigma})^{n-1}}{\int c_{2}(X)(\sigma \bar{\sigma})^{n-1}} .
$$

Thus, $\lambda(L)$ is up to a multiple the value of the quadratic form $f_{X}$ applied to the first Chern class of $L$. 
Proof. This is an immediate application of corollary 4.1 .

Theorem 4.2. Let $X$ be an irreducible symplectic Kähler manifold with symplectic form $\sigma$.

For every line bundle $L$ on $X$, the Euler characteristic of $L$ can be expressed as

$$
\chi(L)=\int_{X} \exp \left(-2 \sum_{k=1}^{\infty} b_{2 k} s_{2 k} T_{2 k}(\sqrt{\lambda(L) / 4+1})\right) .
$$

The right hand side is polynomial in $\lambda(L)$ and thus polynomial in the quadratic form of $c_{1}(L)$.

Proof. We can assume that $\lambda(L) \neq 0$. Since both sides of $(4.6)$ are rational functions in the cohomology class of $\sigma+\bar{\sigma}$ and the equality holds for every irreducible symplectic complex manifold, by a standard argument of hyperkähler geometry, (4.6) remains true, if we substitute $\sigma+\bar{\sigma}$ by an arbitrary cohomology class in $\mathrm{H}^{2}(X)$. Here, we have to substitute $\sigma+\bar{\sigma}$ by $c_{1}(L)$.

Example. Let $\lambda:=\lambda(L)$ be the characteristic value of $L$. Then

$$
\begin{aligned}
& \operatorname{td}\left(\mathcal{T}_{X}(L)\right)=1+\frac{1}{12}\left(c_{2}+\frac{1}{2} c_{2} \cdot \lambda\right) \\
& +\frac{1}{720}\left(3 c_{2}^{2}-c_{4}+\left(\frac{7}{2} c_{2}^{2}-2 c_{4}\right) \lambda+\left(\frac{7}{8} c_{2}^{2}-\frac{1}{2} c_{4}\right) \lambda^{2}\right) \\
& +\frac{1}{30240}\left(\begin{array}{c}
\left(5 c_{2}^{3}-\frac{9}{2} c_{2} c_{4}+c_{6}\right)+\left(\frac{41}{4} c_{2}^{3}-\frac{53}{4} c_{2} c_{4}+\frac{9}{2} c_{6}\right) \lambda \\
+\left(\frac{93}{16} c_{2}^{3}-\frac{33}{4} c_{2} c_{4}+3 c_{6}\right) \lambda^{2}+\left(\frac{31}{32} c_{2}^{3}-\frac{11}{8} c_{2} c_{4}+\frac{1}{2} c_{6}\right) \lambda^{3}
\end{array}\right) \\
& +\cdots,
\end{aligned}
$$

where the $c_{i}$ denote the Chern classes of $X$.

There are (at least) two possibilities to write (4.11) in another form. One is to define "deformed Todd classes" $\operatorname{td}_{\varepsilon}, \varepsilon \in \mathbb{C}$, for arbitrary complex manifolds $X$ :

Definition 18. Let $X$ be an arbitrary complex manifold. We set

$$
\operatorname{td}_{\varepsilon}(X)=\exp \left(\sum_{k=0}^{\infty} t_{k} T_{k}(\varepsilon)\right),
$$

where $t_{k} \in \mathrm{H}^{k, k}$ such that $\ln \operatorname{td}(X)=\sum_{k=0}^{\infty} t_{k}$, i.e. $\operatorname{td}_{1}(X)=\operatorname{td}(X)$.

Applying this definition to our irreducible symplectic Kähler manifold $X$, the equation (4.11) becomes

$$
\chi(L)=\int_{X} \operatorname{td}_{\varepsilon}(X)
$$

with $\varepsilon=\sqrt{\lambda(L) / 4+1}$.

Let us recall some facts about the Grothendieck ring $\mathrm{K}^{0}(X)$ of complex vector bundles over $X$ (see for example [1] $)$. After tensoring with $\mathbb{C}$, the Chern character gives us a ring homomorphism $\mathrm{ch}: \mathrm{K}^{0}(X, \mathbb{C}) \rightarrow \mathrm{H}^{*}(X, \mathbb{C})$ and taking the Todd class gives us a group homomorphism $\mathrm{td}: \mathrm{K}^{0}(X, \mathbb{C}) \rightarrow 1+\mathrm{H}^{*>0}(X, \mathbb{C})$. Furthermore, there are the Adams operations $\psi^{p}: \mathrm{K}^{0}(X, \mathbb{C}) \rightarrow \mathrm{K}^{0}(X, \mathbb{C}), p \in \mathbb{N}$, which are ring homomorphisms with $\psi^{p}(L)=L^{p}$ for every line bundle $L$. The $\psi^{p}$ commute, and we can write $\mathrm{K}^{0}(X, \mathbb{C})=\bigoplus_{k=0}^{\infty} \operatorname{Gr}^{2 k} \mathrm{~K}^{0}(X, \mathbb{C})$ such that $\operatorname{Gr}^{2 k} \mathrm{~K}^{0}(X, \mathbb{C})$ is the eigenspace of $\psi^{p}$ for $p \geq 2$ to the eigenvalue $p^{k}$. Using this grading on $\mathrm{K}^{0}(X, \mathbb{C})$, the Chern character becomes a homomorphism of graded rings. 
If $M=\bigoplus_{i \in \mathbb{N}_{0}} M_{i}$ is a graded $R$-module, $R$ a commutative ring and $\left(\lambda_{i}\right)_{i \in \mathbb{N}_{0}}$ is a sequence in $R$, we define a morphism of graded modules $\left(\lambda_{i}\right)_{i \in \mathbb{N}_{0}} \cdot: M \rightarrow M, m \in$ $M_{i} \mapsto \lambda_{i} \cdot m$. This definition will be applied to $\mathrm{K}^{0}(X, \mathbb{C})$ :

Definition 19. For every complex number $\varepsilon \in \mathbb{C}$ and complex manifold $X$, one defines a homomorphism

$$
\phi_{\varepsilon}: \mathrm{K}^{0}(X, \mathbb{C}) \rightarrow \mathrm{K}^{0}(X, \mathbb{C}), E \mapsto\left(T_{i}(\varepsilon)\right)_{i \in \mathbb{N}_{0}} \cdot E
$$

Applying this definition to our irreducible symplectic Kähler manifold $X$, the equation (4.11) becomes

$$
\chi(L)=\int_{X} \operatorname{td}\left(\phi_{\varepsilon}\left(\mathcal{T}_{X}\right)\right)
$$

with $\varepsilon=\sqrt{\lambda(L) / 4+1}$.

\section{Generalized Kummer Varieties.}

5.1. Basic Facts. Generalized Kummer varieties were introduced by Beauville in [3] as the second series of examples of irreducible symplectic manifolds. We briefly recall their construction and some basic properties of these varieties.

We start with an abelian surface $A$. Let $A^{(n)}:=A^{n} / \mathfrak{S}_{n}$ be the $n$-fold symmetric product, denote by $A^{[n]}$ the $n$-th Hilbert scheme of points on $A$, and let $\rho: A^{(n)} \longrightarrow$ $A^{[n]}$ be the Hilbert-Chow-morphism. Since summation $A^{n} \longrightarrow A$ is symmetric, it factors over $A^{(n)}$, and by composing with $\rho$ one gets the "summation" morphism $s: A^{[n]} \longrightarrow A$. Now the $(n-1)$-th Kummer variety $K^{n-1} A$ is defined as the fiber over 0 of the summation morphism $s$. As was shown by Beauville, $K^{n-1} A$ is an irreducible symplectic variety of dimension $2(n-1)$. In order to fix notations, we briefly show that the $(n-1)$-th Kummer variety is smooth.

This can be seen as follows: Denote the translation operation on $A^{[n]}$ by an element $a \in A$ by $t_{a}$. Considering $A$ acting on $A^{[n]}$ via $t_{a}$ while acting on $A$ via $t_{n a}$, one sees that $s$ is an equivariant morphism. Since $A$ acts transitively on itself, all fibers are isomorphic. (In particular, the definition of $K^{n-1} A$ is independent of the choice of $0 \in A$.) Since there are smooth fibers, the Kummer variety is smooth.

Actually, the fibration $A^{[n]} \stackrel{s}{\longrightarrow} A$ is isotrivial, i.e. one has the following Cartesian diagram, where $n$ denotes the morphism "multiplication by $n$ " $A \stackrel{\Delta}{\rightarrow} A^{n} \stackrel{\Sigma}{\rightarrow} A$ :

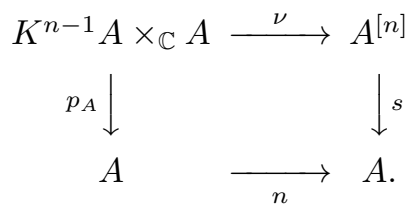

In terms of closed points, the fibre product is $\left\{(\xi, a) \in A^{[n]} \times A \mid s(\xi)=n a\right\}$. This is isomorphic to $K^{n-1} A \times A$ via $(\xi, a) \mapsto\left(t_{-a}(\xi), a\right)$. Therefore, on closed points the morphism $\nu$ in the above diagram is given by $(\xi, a) \mapsto t_{a}(\xi)$.

Example. The classical Kummer surface.

The easiest example - and the reason for the terminology 'generalized Kummer varieties' - is the classical Kummer surface. In the classical context this surface is constructed as follows (for details, cf. e.g. [4]):

One starts with an Abelian surface $A$ and considers the singular quotient $A / \sim$ by the involution $(-1)_{A}$. The singularities are the images of the 16 two-division points. The desingularization, which we again denote by $K^{1} A$, is the classical Kummer suface which is an K3 surface. 
Alternatively one can first blow up the 16 points of order 2 of $\mathrm{A}$ and let $K^{1} A$ be the quotient of the induced involution on the blown up surface $\hat{A}$. In other words, one has the following diagram:

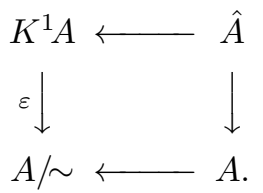

$K^{1} A$ can be identified with the fiber over 0 of $s: A^{[2]} \longrightarrow A$ as follows:

In the case $n=2$ the Hilbert Chow morphism $\rho_{2}: A^{[2]} \longrightarrow A^{(2)}$ is simply the blow-up of the diagonal $\Delta \subset A^{(2)}$ (cf. [8]). Denote by $\tilde{\Delta}: A \longrightarrow A^{(2)}$ the morphism induced from the two isomorphisms $\operatorname{id}_{A}$ and $(-1)_{A}$. On closed points, $\tilde{\Delta}$ is given by $a \mapsto(a,-a)$. By definition of $A^{(2)}$ and $A / \sim$, it descends to an morphism $A / \sim \longrightarrow A^{(2)}$, which we again denote by $\tilde{\Delta}$. From the universal property of the fibre product, we get an isomorphism $A / \sim \longrightarrow \Sigma^{-1}(0)$.

So, we have the following diagram:

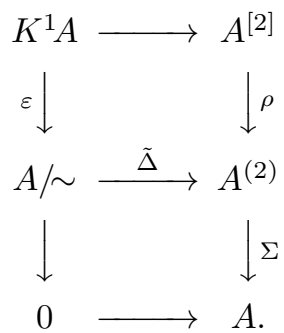

This shows that $\varepsilon=\left.\rho\right|_{K^{1} A}$ and that the two descriptions of $K^{1} A$ coincide.

5.2. Explicit Hirzebruch-Riemann-Roch for $K^{n} A$. In this section we will prove the following Hirzebruch-Riemann-Roch formula formula without using RozanskyWitten classes in a purely algebro-geometric way.

Theorem 5.1. Let $L$ be a line bundle on $K^{n} A$. The Euler characteristics of $L$ is given by

$$
\chi(L)=(n+1)\left(\begin{array}{c}
\frac{(n+1)}{4} \lambda(L)+n \\
n
\end{array}\right),
$$

where $\lambda(L)$ is the characteristic value of $L$ defined in section 4.9 .

We will prove the theorem as follows: First, observe that since the HirzebruchRiemann-Roch formula has the form $\chi(L)=\sum a_{2} i /(2 i) ! q_{X}(L)^{i}$ with universal coefficients $a_{i}$, it is actually enough to consider a single line bundle $L$ with $\chi(L) \neq 0$ and computing the coefficients by calculating $\chi\left(L^{m}\right)$. We will prove the theorem using special line bundels $K^{n} L$ on $K^{n} A$, which are constructed from an invertible sheaf $L \in \operatorname{Pic}(A)$ as follows:

In a first step define $S^{(n+1)} L:=\pi_{*}\left(L^{\bigotimes(n+1)}\right)^{\mathfrak{S}_{(n+1)}}$ the $\mathfrak{S}_{(n+1)}$-symmetrized line bundle on $A^{(n+1)}$. Here, $\pi: A^{n+1} \rightarrow A^{(n+1)}$ is the projection and $L^{\bigotimes n}$ denotes the sheaf $\bigotimes_{1=1}^{n} \operatorname{pr}_{i}^{*} L$. Now define $L_{(n+1)}:=\rho_{(n+1)}^{*} S^{(n+1)} L \in \operatorname{Pic}\left(A^{[n+1]}\right)$. We are interested in the cohomology of the restricted bundle $K^{n} L:=\left.L_{(n+1)}\right|_{K^{n} A}$ on the $n$-th Kummer variety.

One knows that for such bundles the quadratic form $f_{X}\left(c_{1}\left(K^{n} L\right)\right)$ coincides up to a positive scalar factor - with the intersection pairing $c_{1}(L)^{2}$ on $\mathrm{H}^{2}(A, \mathbb{Z})$ (cf. 3]). If we normalize the quadratic form by defining $q\left(K^{n} L\right):=c_{1}(L)^{2}$, our theorem follows from the following 
Lemma 5.2. The Euler characteristics of $L \in \operatorname{Pic}(A)$ and of $K^{n} L \in \operatorname{Pic}\left(K^{n} A\right)$ are related by

$$
\chi\left(K^{n} L\right)=(n+1)\left(\begin{array}{c}
\frac{c_{1}(L)^{2}}{2}+n \\
n
\end{array}\right) .
$$

Proof of the Theorem. Using the lemma above the theorem is proven if we show that $q=\frac{n+1}{2} \lambda$.

By definition of $K^{n} L$ and $q$ we get

$$
q\left(\left(K^{n} L\right)^{\otimes m}\right)=q\left(K^{n}\left(L^{\otimes m}\right)\right)=m^{2} q\left(K^{n} L\right)
$$

for every $m \in \mathbb{N}$.

Now HRR in its classical form - using $c_{1}(X)=0$ - gives

$\chi\left(K^{n} L^{\otimes m}\right)=m^{2 n} \int \operatorname{ch}\left(K^{n} L\right)+m^{2 n-2} \int \frac{c_{2}(X)}{12} \operatorname{ch}\left(K^{n} L\right)+$ terms of lower degree,

whereas our formula in lemma 5.2 reads

$$
\chi\left(K^{n} L^{\otimes m}\right)=m^{2 n} \frac{n+1}{2^{n} n !} q\left(K^{n} L\right)^{n}+m^{2 n-2} \frac{(n+1)^{2} n}{2^{n} n !} q\left(K^{n} L\right)^{n-1}+\text { told. }
$$

Comparing coefficients, we find

$$
\lambda\left(K^{n} L\right)=\frac{24 n \int \operatorname{ch}\left(K^{n} L\right)}{\int c_{2}(X) \operatorname{ch}\left(K^{n} L\right)}=\frac{2}{n+1} q\left(K^{n} L\right) .
$$

Since both $\lambda$ and $q$ are positive multiples of $f_{X}$ and the coefficients of the HRR formula as a polynomial in the quadratic form are independent of the line bundle considered, the theorem follows.

The rest of this section is dedicated to the proof of lemma 5.2. To simplify notations we will prove the assertion for $K^{n-1} L \in \operatorname{Pic}\left(K^{n-1} A\right)$.

In the next lemma we will compute the Euler characteristic of the symmetrized line bundle $S^{n} L$.

Lemma 5.3. Let $L$ be a line bundle on $A$. Then one has

$$
\chi\left(S^{n} L\right)=\left(\begin{array}{c}
\chi(L)+n-1 \\
n
\end{array}\right)
$$

Proof. Let $L, H \in \operatorname{Pic}(A)$. One has

$$
S^{n}(L \otimes H)=\pi_{*}\left((L \otimes H)^{\bigotimes n}\right)^{\mathfrak{S}_{n}}=\pi_{*}\left(L^{\bigotimes n} \otimes H^{\bigotimes n}\right)^{\mathfrak{S}_{n}},
$$

and since the action of the symmetric group does not flip the factors $L^{\bigotimes n}$ and $H^{\bigotimes n}$, and for an arbitrary line bundle $M$ on $A$ we have $\pi^{*} S^{n} M=M^{\bigotimes n}$, we get

$$
S^{n}(L \otimes H)=\pi_{*}\left(L^{\bigotimes n} \otimes \pi^{*} S^{n} H\right)^{\mathfrak{S}_{n}}=\left(\pi_{*}\left(L^{\bigotimes n}\right) \otimes S^{n} H\right)^{\mathfrak{S}_{n}}=S^{n} L \otimes S^{n} H
$$

Now, if $H$ is an ample invertible sheaf, $H^{\bigotimes n}$ is also ample and so is $S^{n} H$, because $\pi$ is a finite surjective morphism and $\pi^{*} S^{n} H=H^{\bigotimes n}$ is ample. Let $N \in \mathbb{N}$ be large enough that both $L \otimes H^{N}$ and $S^{n}\left(L \otimes H^{N}\right)=S^{n} L \otimes\left(S^{n} H\right)^{N}$ have no higher cohomology such that $\chi\left(S^{n}\left(L \otimes H^{N}\right)\right)=h^{0}\left(S^{n}\left(L \otimes H^{N}\right)\right)$.

For the global sections of an symmetrized line bundle $S^{n} M$, one has the isomorphism

$$
\mathrm{H}^{0}\left(A^{(n)}, S^{n} M\right) \simeq \mathrm{H}^{0}\left(A^{n}, M^{\bigotimes n}\right)^{\mathfrak{S}_{n}}=\left(\mathrm{H}^{0}(A, M)^{\otimes n}\right)^{\mathfrak{S}_{n}} \simeq \operatorname{Sym}^{n} \mathrm{H}^{0}(A, M) .
$$

Now replacing $M$ by $L \otimes H^{N}$, we find

$$
\chi\left(S^{n}\left(L \otimes H^{N}\right)\right)=\left(\begin{array}{c}
\chi\left(L \otimes H^{N}\right)+n-1 \\
n
\end{array}\right) \quad \text { for all } N \gg 0 .
$$


Since $N \mapsto \chi\left(S^{n}\left(L \otimes H^{N}\right)\right)=\chi\left(S^{n} L \otimes\left(S^{n} H\right)^{N}\right)$ is a polynomial in $N$, evaluation in $N=0$ proves the lemma.

Consider the Hilbert-Chow morphism $\rho: A^{[n]} \longrightarrow A^{(n)}$. Since $\rho$ is a birational proper morphism of normal varieties one has $\rho_{*} \mathcal{O}_{A^{[n]}}=\mathcal{O}_{A^{(n)}}$. Furthermore, $A^{(n)}$ has rational singularities as quotient of a smooth variety by a finite group (cf. [13]). Therefore its resolution $\rho$ satisfies $R^{j} \rho_{*} \mathcal{O}_{A^{[n]}}=0$ for $j>0$. Using the Leray spectral sequence one gets

$$
\mathrm{H}^{i}\left(S^{n} L\right)=\mathrm{H}^{i}\left(\rho^{*} S^{n} L\right)
$$

So we have proven the following

Proposition 11. For a line bundle $L \in \operatorname{Pic}(A)$ one has

$$
\chi\left(L_{n}\right)=\left(\begin{array}{c}
\chi(L)+n-1 \\
n
\end{array}\right)
$$

Remark. This result is proven by a somewhat different method in [7].

Next we will attack the cohomology of the restricted bundle $K^{n-1} L$. The first step in this direction is the following

Lemma 5.4. In the notation of diagram (5.1), one has $\nu^{*} L_{n}=K^{n-1} L \otimes L^{n}$.

Proof. The splitting of the sheaf $\nu^{*} L_{n}$ follows from the seesaw principle (cf. [14): For fixed $a \in A$ we have seen that the restricted morphism $\left.\nu\right|_{K^{n-1} A \times\{a\}}$ is the isomorphism wich maps $K^{n-1} A$ to the fiber of the summation morphism $s$ over the point $n a$. Since $K^{n-1} A$ is simply connected, its Picard group is discrete and it follows that $\left.\left.\nu^{*} L_{n}\right|_{K^{n-1} A \times\{a\}} \simeq p_{K^{n-1} A}^{*} K^{n-1} L\right|_{K^{n-1} A \times\{a\}}$.

Therefore $\nu^{*} L_{n}$ is of the form $K^{n-1} L \otimes L_{2}$ with $L_{2} \in \operatorname{Pic}(A)$, and we can compute the component $L_{2}$ by considering the restrictions of $\nu$ to $\left\{\xi_{0}\right\} \times A$.

Now complete diagram (5.1) as follows

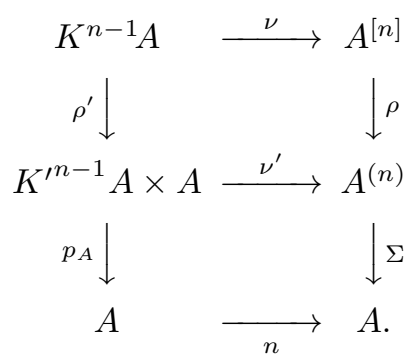

Here $K^{\prime n-1} A$ denotes the "singular Kummer", the fiber over 0 of the addition morphism $\Sigma: A^{(n)} \longrightarrow A, \rho^{\prime}=\left.\rho\right|_{K^{\prime n-1} A} \times i d_{A}$ its desingularization, and $\nu^{\prime}$ is defined analogously to $\nu$.

Now consider a point $\xi_{0} \in K^{n-1} A$ over $(0, \ldots, 0) \in A^{(n)}$. Instead of computing the sheaf $\left.\nu^{*} L_{n}\right|_{\left\{\xi_{0}\right\} \times A}$ we equivalently compute $\left.\rho^{\prime *} \nu^{\prime *} S^{n} L\right|_{\left\{\xi_{0}\right\} \times A}=L^{n}$.

The next lemma describes the structure of the direct image of $\mathcal{O}_{A}$ under the $n^{4}$-fold covering $A \stackrel{n}{\longrightarrow} A$.

Lemma 5.5. The direct image $n_{*} \mathcal{O}_{A}$ of the structure sheaf of $A$ splits into a direct sum of line bundles $L_{i}, i \in A[n]$, indexed by the $n$-torsion points of $A$ and with $L_{0}=\mathcal{O}_{A}$ and $c_{1}\left(L_{i}\right)_{\mathbb{Q}}=0 \in \mathrm{H}^{2}(A, \mathbb{Q})$ for all $i$.

Proof. The splitting of $n_{*} \mathcal{O}_{A}$ is a well known fact, cf. 14, $\S 7$. The triviality of their first rational Chern classes follows from the relation:

$$
L_{i}^{n}=L_{i n}=L_{0}=\mathcal{O} \text {. }
$$


It follows that $n c_{1}\left(L_{i}\right)=0$, which proves the lemma.

Now we have collected all necessary ingredients for the proof of lemma 5.2.

Proof of the lemma. We start again with a line bundle $L$ on $A$ that we twist with a sufficiently ample bundle $H^{N}$. By abuse of notation we denote the resulting bundle by $L$ again. By construction the symmetrized bundle $S^{n} L$ is still ample such that the invertible sheaf $L_{n}$ on $A^{[n]}$ as a pull back along the birational morphism $\rho$ is nef and big.

The same argument shows that the line bundle $K^{n-1} L$ is nef and big: Using the notations of diagram (5.4) the bundle $K^{\prime n-1} L:=\left.\nu^{\prime *} S^{n} L\right|_{K^{\prime n-1} A}$ is ample and $K^{n-1} L=\rho^{\prime *} K^{\prime n-1} L$ is big and nef.

So by the Kawamata-Viehweg vanishing theorem (cf. [18]), we have

$$
\chi\left(K^{n-1} L\right)=h^{0}\left(K^{n-1} A, K^{n-1} L\right)
$$

On the one hand, we have due to the Künneth formula

$$
\mathrm{H}^{0}\left(\nu^{*} L_{n}\right)=\mathrm{H}^{0}\left(K^{n-1} L \otimes L^{n}\right)=\mathrm{H}^{0}\left(K^{n-1} L\right) \otimes \mathrm{H}^{0}\left(L^{n}\right) .
$$

On the other hand, since $\nu$ is finite and $n$ is a flat morphism, we can compute $\mathrm{H}^{0}\left(\nu^{*} L_{n}\right)$ alternatively

$$
\mathrm{H}^{0}\left(\nu^{*} L_{n}\right)=\mathrm{H}^{0}\left(L_{n} \otimes \nu_{*} \mathcal{O}_{K^{n-1} A \times A}\right)=\mathrm{H}^{0}\left(L_{n} \otimes s^{*} n_{*} \mathcal{O}_{A}\right)=\mathrm{H}^{0}\left(L_{n} \otimes \bigoplus_{i \in A[n]} s^{*} L_{i}\right) .
$$

This shows that

$$
h^{0}\left(K^{n-1} A \times A, \nu^{*} L\right)=\sum_{i \in A[n]} h^{0}\left(A^{[n]}, L_{n} \otimes L_{i}^{\prime}\right)
$$

with $L_{i}^{\prime}:=s^{*} L_{i}$.

Since the sheaf $L_{n} \otimes L_{i}^{\prime}$ is still nef and big, the vanishing theorem of Kawamata and Viehweg implies that $h^{0}\left(A^{[n]}, L_{n} \otimes L_{i}^{\prime}\right)$ equals the Euler characteristic of this line bundle. Therefore, using the classical Hirzebruch-Riemann-Roch theorem on $A^{[n]}$ we have

$$
\begin{aligned}
h^{0}\left(\nu^{*} L_{n}\right) & =\sum_{i \in A[n]} \chi\left(A^{[n]}, L_{n} \otimes L_{i}^{\prime}\right) & & \\
& =\sum_{i \in A[n]} \int \operatorname{ch}\left(L_{n} \otimes L_{i}^{\prime}\right) \operatorname{td}\left(A^{[n]}\right) & & \\
& =n^{4} \int \operatorname{ch}\left(L_{n}\right) \operatorname{td}\left(A^{[n]}\right), & & \text { since } c_{1}\left(L_{i}^{\prime}\right)_{\mathbb{Q}}=s^{*} c_{1}\left(L_{i}\right)_{\mathbb{Q}}=0 \\
& =n^{4} \chi\left(A^{[n]}, L_{n}\right) & & \\
& =n^{4} \operatorname{dim}\left(S^{n} \mathrm{H}^{0}(L)\right) & & \text { due to } 5.3) .
\end{aligned}
$$

Now combining the computations - and noting that $h^{0}\left(A, L^{n}\right) \neq 0$ - we find

$$
\begin{aligned}
\chi\left(K^{n-1} A, K^{n-1} L\right) & =h^{0}\left(K^{n-1} L\right)=\frac{h^{0}\left(\nu^{*} L_{n}\right)}{h^{0}\left(A, L^{n}\right)}=\frac{\left.n^{4} \frac{\frac{c_{1}(L)^{2}}{2}+n-1}{n}\right)}{n^{2} \frac{c_{1}(L)^{2}}{2}} \\
& =n\left(\begin{array}{c}
\frac{c_{1}(L)^{2}}{2}+n-1 \\
n-1
\end{array}\right) .
\end{aligned}
$$

Once again - considering the formula as a polynomial in $N$ and evaluating in $N=0$ - the formula holds for a general line bundle $L$. 
Example. In the case of the Kummer surface $K^{1} A$, the above formula gives back the classical Riemann-Roch formula for K3 surfaces:

Remember the diagram

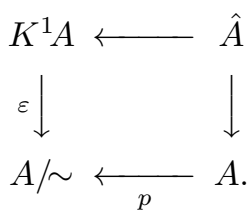

Let us start with an symmetric line bundle $L$ on $A$, i.e. $L=p^{*} L^{\prime}$ with $L^{\prime} \in$ $\operatorname{Pic}(A / \sim)$. Then $L$ induces a line bundle $M=\varepsilon^{*} L^{\prime}$ on $K^{1} A$. Write $K^{1} L=$ $\rho^{*} \pi_{*}(L \otimes L)^{\mathfrak{S}_{2}}$, as usual. Then one has $K^{1} L=M^{2}$ :

Considering diagram (5.2), it suffices to show that $\tilde{\Delta}^{*} S^{2} L=L^{\prime 2}$. But this is clear from the definition of $\Delta$ and $L=p^{*} L^{\prime}$.

Now, our HRR formula gives

$$
\chi\left(K^{1} L\right)=2\left(\begin{array}{c}
\frac{c_{1}(L)^{2}}{2}+1 \\
1
\end{array}\right)=c_{1}(L)^{2}+2
$$

Using that $\varepsilon$ is birational, $p$ is generically $2: 1$ and the equality $K^{1} L=M^{2}$, one finds

$$
\chi\left(K^{1} L\right)=\frac{c_{1}\left(K^{1} L\right)^{2}}{2}+2
$$

which is the classical Riemann-Roch formula for the K3 surface $K^{1} A$.

\section{Characteristic numbers of $K^{n} A$}

In his Ph.D. thesis (cf. [16]), Sawon calculated all Chern numbers for the generalized Kummer varieties $K^{n} A$ with $n \leq 4$.

His method is to use the usual Hirzebruch-Riemann-Roch formula to express the $\chi_{y}$-genus, which has been calculated by Göttsche and Soergel (see [9]) in terms of characteristic numbers. This gives him $k$ independent relations between the Chern numbers on a generalized Kummer variety. These are enough relations for $n \leq 3$ to determine the values of the Chern numbers. For $n=4$, one further relation is needed. Sawon managed to calculate the characteristic numbers $\int_{K^{n} A} \sqrt{\operatorname{td}\left(K^{n} A\right)}$, which gives him another relation on the generalized Kummer variety $K^{4} A$, thus enabling him to calculate the Chern numbers of $K^{4} A$.

Of course, we can use our formulas to derive relations beetween the Chern numbers of the generalized Kummer varieties. These relations together with the relations given by the known value of the $\chi_{y}$-genus leads to all Chern numbers of $K^{n} A$, for $n \leq 5$.

Let $L$ be a line bundle with non-vanishing quadratic form on a generalized Kummer variety $K^{n} A$ of complex dimension $2 n$. Let $\lambda:=\lambda(L)$ be the characteristic value (as in section 4.2) of $L$ on $K^{n} A$. So, $\lambda\left(L^{\otimes m}\right)=m^{2} \lambda$ for all $m \in \mathbb{N}_{0}$.

According to our previous results, we have

$$
\int_{K^{n} A} \operatorname{td} \sqrt{m^{2} \lambda / 4+1}\left(K^{n} A\right)=\chi\left(L^{\otimes m}\right)=(n+1)\left(\begin{array}{c}
\frac{1}{4} m^{2} \lambda(n+1)+n \\
n
\end{array}\right)
$$

for $m \in \mathbb{N}_{0}$. Since both sides are polynomial in $m$, we can compare the coefficients of $m$, which gives us no more than $\left\lfloor\frac{n+1}{2}\right\rfloor$ independent expressions for some characteristic numbers of $K^{n} A$ since the coefficients on the left are given by Chern 
numbers. For example, the leading coefficients yield

$$
\int_{K^{n} A} \sqrt{\operatorname{td}\left(K^{n} A\right)}=\frac{(n+1)^{n+1}}{4^{n} n !},
$$

a formula which has already been found by Sawon ([16]). The comparision of coefficients also yields

$$
\int_{K^{n} A} \operatorname{td}\left(K^{n} A\right)=n+1,
$$

which holds on every irreducible symplectic Kähler manifold of complex dimension $2 n$.

We also have to investigate Hirzebruch's $\chi_{y}$-genus, which is given by

$$
\chi_{y}\left(K^{n} A\right)=\chi\left(\bigwedge_{y} \Omega_{X}\right)=\sum_{p=0}^{2 n} y^{p} \int_{X} \operatorname{td}(X) \operatorname{ch}\left(\Omega_{X}^{p}\right) .
$$

Here, $\bigwedge_{y} \Omega_{X}=\sum_{p=0}^{2 n} y^{p} \Omega_{X}^{p} \in \mathrm{K}^{0}\left(K^{n} A\right)[y]$. Apparently, $\chi_{y}\left(K^{n} A\right)$ can be expressed by the Hodge numbers $h^{*, *}(X)$ of $K^{n} A$, which have been calculated by Göttsche and Soergel (see [9]). It is

$$
\chi_{y}\left(K^{n} A\right)=(n+1) \sum_{d \mid(n+1)} d^{3}\left(1-y+y^{2}-\cdots+(-y)^{\frac{k+1}{d}-1}\right)^{2}(-y)^{k+1-\frac{k+1}{d}} .
$$

Using (6.4), this yields at most $n$ further independent expressions for Chern numbers of $K^{n} A$ (cf. [16]). One of these expressions is again (6.3), so summing up, we have at most $\left[\frac{n+1}{2}\right]+n-1$ linear independent equations for the Chern numbers on $K^{n} A$.

We used the computer algebra system Maple to solve the linear relations for the seven Chern numbers on $K^{5} A$ and arrived at the following table:

\begin{tabular}{c|r} 
Chern number & Evaluated on $K^{5} A$ \\
\hline$c_{2}^{5}$ & 84478464 \\
$c_{2}^{3} c_{4}$ & 26220672 \\
$c_{2}^{2} c_{6}$ & 3141504 \\
$c_{2} c_{8}$ & 142560 \\
$c_{2} c_{4}^{2}$ & 8141472 \\
$c_{4} c_{6}$ & 979776 \\
$c_{10}$ & 2592
\end{tabular}

To calculate the ten Chern numbers of $K^{6} A$, our methods are not sufficient since they lead only to eight independent relations.

\section{REFERENCES}

1. Michael. F. Atiyah, K-theory, W. A. Benjamin, Inc., 1967.

2. Dror Bar-Natan, On the Vassiliev knot invariants, Topology 34 (1995), no. 2, 423-472.

3. Arnaud Beauville, Variétés Kähleriennes dont la première classe de Chern est nulle, J. Differential Geom. 18 (1983), no. 4, 755-782 (1984).

4. Arnaud Beauville, Complex algebraic surfaces, second ed., Cambridge University Press, Cambridge, 1996.

5. S. V. Chmutov and S. V. Duzhin, A lower bound for the number of Vassiliev knot invariants, Topology Appl. 92 (1999), no. 3, 201-223.

6. Oliver T. Dasbach, On the combinatorial structure of primitive vassiliev invariants, II, J. Combin. Theory 81 (1998), no. 2, 127-139.

7. Geir Ellingsrud, Lothar Göttsche, and Manfred Lehn, On the cobordism class of the Hilbert scheme of a surface, arXiv:math.AG/9904095, to appear in J. Alg. Geom.

8. John Fogarty, Algebraic families on an algebraic surface, Amer. J. Math 90 (1968), 511-521. 
9. Lothar Göttsche and Wolfgang Soergel, Perverse sheaves and the cohomology of Hilbert schemes of smooth algebraic surfaces, Math. Ann. 296 (1993), no. 2, 235-245.

10. Nigel Hitchin and Justin Sawon, Curvature and characteristic numbers of hyperkähler manifolds, arXiv:math.DG/9908114, to appear in Duke Mathematical Journal.

11. Daniel Huybrechts, Compact hyper-Kähler manifolds: basic results, Invent. Math. 135 (1999), no. $1,63-113$.

12. Mikhail Kapranov, Rozansky-Witten invariants via Atiyah classes, Compos. Math. 115 (1999), no. 1, 71-113.

13. János Kollár and Shigefumi Mori, Birational geometry of algebraic varieties, Cambridge University Press, Cambridge, 1998.

14. David Mumford, Abelian varieties, Published for the Tata Institute of Fundamental Research, Bombay, 1970, Tata Institute of Fundamental Research Studies in Mathematics, No. 5.

15. Lev Rozansky and Edward Witten, Hyper-Kähler geometry and invariants of three-manifolds, Selecta Math. (N.S.) 3 (1997), no. 3, 401-458.

16. Justin Sawon, Rozansky-Witten invariants of hyperkähler manifolds, Ph.D. thesis, University of Cambridge, October 1999.

17. Dylan P. Thurston, Wheeling: A diagrammatic analogue of the Duflo isomorphism, Ph.D. thesis, University of California at Berkeley, arXiv:math.QA/0006083, Spring 2000.

18. Eckart Viehweg, Vanishing theorems, J. Reine Angew. Math. 335 (1982), 1-8.

Mathematisches Institut DeR Univ. ZU Köln, Weyertal 86-90, 50931 Köln, Germany E-mail address: mbritze@mi.uni-koeln.de, mail@marc-nieper.de 